\title{
ACCURACY MEASURES AND FOURIER ANALYSIS FOR THE FULL MULTIGRID ALGORITHM*
}

\author{
CARMEN RODRIGO ${ }^{\dagger}$, FRANCISCO J. GASPAR $^{\ddagger}$, CORNELIS W. OOSTERLEE ${ }^{\S}$, AND \\ IRAD YAVNEH
}

\begin{abstract}
The full multigrid (FMG) algorithm is often claimed to achieve so-called discretization-level accuracy. In this paper, this notion is formalized by defining a worst-case relative accuracy measure, denoted $E_{F M G}^{\ell}$, which compares the total error of the $\ell$-level FMG solution against the inherent discretization error. This measure can be used for tuning algorithmic components so as to obtain discretization-level accuracy. A Fourier analysis is developed for estimating $E_{F M G}^{\ell}$, and the resulting estimates are confirmed by numerical tests.
\end{abstract}

Key words. full multigrid algorithm, discretization error, local Fourier analysis, FMG measure

AMS subject classifications. $65 \mathrm{~N} 55,65 \mathrm{~F} 10$

DOI. $10.1137 / 100786101$

1. Introduction. Modern numerical methods for partial differential equations (PDE) often exploit hierarchies of computational grids of various resolutions to accelerate iterative solution procedures. Typically, the problem is initially discretized and solved on a very coarse grid, and this solution is interpolated to a finer grid, where it serves as an initial approximation. Several iterations of some numerical algorithm are employed, and the result is interpolated to a still finer grid, and so on, until an approximate solution is obtained on the target grid. The technique, known as nested iteration, was suggested by Kronsjö and Dahlquist in [9, 10], where it was combined with the Successive Over-Relaxation (SOR) method for the Laplace equation on a unit square. Later, nested iteration was combined with multigrid computational techniques $[2,6,15]$, yielding the so-called full multigrid (FMG) algorithm. In this well-known approach, the iterative solver is a multigrid cycle which employs the very same grid hierarchy to greatly accelerate the convergence of a basic iterative solver (relaxation). Typically, just one or two multigrid cycles are applied at each level of the hierarchy, resulting in a linear computational complexity. The key question then is whether the solution obtained by this algorithm is "sufficiently accurate." This question is at the focus of this paper.

As the discrete solution approximates the continuous solution only up to discretization accuracy (formulated below), the goal of the FMG algorithm should be to yield a numerical solution whose error is comparable to the discretization error. It is not worthwhile to solve the discrete problem more accurately by investing more computational work. Indeed, if more computational work is to be invested, it should

*Received by the editors February 16, 2010; accepted for publication (in revised form) August 19, 2010; published electronically October 12, 2010. This research was partially supported by FEDER/MCYT Projects MTM2007-63204 and the DGA (Grupo consolidado PDIE).

http://www.siam.org/journals/sisc/32-5/78610.html

${ }^{\dagger}$ Centro Universitario de la Defensa. Academia General Militar. Ctra. de Huesca s/n, 50090, Zaragoza, Spain (carmenr@unizar.es).

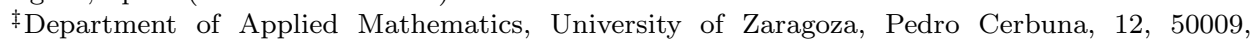
Zaragoza, Spain (fjgaspar@unizar.es).

$\S$ CWI, Centrum Wiskunde \& Informatica, Science Park 123, 1098 XG Amsterdam, the Netherlands, and Delft University of Technology, the Netherlands (c.w.oosterlee@cwi.nl).

IDepartment of Computer Science, Technion-Israel Institute of Technology, Haifa 32000, Israel (irad@cs.technion.ac.il). 
be aimed at reducing the discretization error itself, for example, by appealing to a yet finer target grid. For some types of problems, the common lore states that one or two multigrid cycles are sufficient to reach such discretization accuracy. In this sense, FMG is considered to be asymptotically optimal; that is, the number of arithmetic operations required is proportional to the number of grid points, with only a small constant of proportionality.

In practice, it may be quite difficult to assess whether the FMG solution indeed yields discretization-level accuracy. The residual norm, which is a common measure of accuracy, is not directly useful for this task. FMG has received relatively little attention in terms of analysis. Hackbusch reserves a chapter to nested iteration in his book [6], and in [14] a quantitative analysis is performed for multigrid W-cycles, where a two-level convergence factor of 0.1 guarantees the optimal convergence of FMG given one extra multigrid iteration on the finest grid level. Such a convergence factor represents a severe criterion, which is not easily achieved, for example, for complicated systems of equations.

Our aim here is to develop a local Fourier analysis (LFA) framework for FMG. We wish to develop a tool which yields, a priori, valuable insights into the various components of the FMG algorithm and their effect on the final relative accuracy. LFA, introduced by Brandt in 1977 [2] as local mode analysis, is a useful technique for choosing suitable components for multigrid algorithms. In particular, the smoother, the transfer operators, the number of pre- and post-smoothing steps, the type of multigrid cycle (e.g., V-cycle or W-cycle) have to be selected for each concrete problem. From a practical point of view, this analysis is helpful because it provides realistic quantitative estimates of the asymptotic multigrid convergence factor. Wienands and Joppich [18] provide a useful software tool for experimenting with Fourier analysis. Recent advances in LFA analysis include LFA for multigrid as a preconditioner [17], for triangular meshes [5], for optimal control problems [1], and for discontinuous Galerkin discretizations [8]. A first notion of FMG analysis appeared in the "Guide to Multigrid Development" by Brandt [3], but, to our knowledge, it has never been formalized or worked out in detail in the context of LFA.

The organization of the paper is as follows. In section 2, basic definitions and notations for the operators of the FMG algorithm are presented. In particular, a suitable indicator of the performance of the FMG algorithm, the so-called FMG accuracy measure, is defined. Section 3 is devoted to the LFA analysis employed to estimate the FMG accuracy measure for $k$ grid levels. Also, the analysis is validated by introducing the concept of the worst-case right-hand side analysis. Finally, some numerical experiments are presented in section 4 to demonstrate the potential of the $k$-level FMG analysis, followed by conclusions.

2. Definitions and notations. In order to describe the full multigrid algorithm, a general boundary value problem on an open bounded domain $\Omega \subset \mathbb{R}^{d}$ is considered,

$$
\mathcal{L} u=f, \text { in } \Omega,
$$

with appropriate boundary conditions. Let $L_{1} u_{1}=f_{1}$ be a discretization of problem (2.1) on a (fine) target grid $\Omega_{1}$, where the grid function $f_{1}$ is given by $f_{1}=\mathcal{J}_{1} f$, with $\mathcal{J}_{1}$ denoting a transfer operator from the continuum to the fine grid. Throughout this paper, $\mathcal{J}_{1}$ is defined as simple evaluation (injection), although, in general, local averaging may be applied and included in the analysis. A sequence of coarser grids, $\Omega_{2}, \Omega_{3}, \ldots, \Omega_{\ell}$, and appropriate discrete problems $L_{k} u_{k}=f_{k}$ on $\Omega_{k}, k=2, \ldots, \ell$, are 
introduced. Here, $L_{k}$ is a suitable approximation of the fine grid discrete operator $L_{1}$ on $\Omega_{k}$, and $f_{k}$ is given by $f_{k}=J_{k-1}^{k} f_{k-1}, k=2, \ldots, \ell$, with $J_{k-1}^{k}: \mathcal{G}\left(\Omega_{k-1}\right) \rightarrow \mathcal{G}\left(\Omega_{k}\right)$ some restriction operator, where $\mathcal{G}\left(\Omega_{k}\right)$ denotes the set of grid functions defined on $\Omega_{k}$.

As commented before, the FMG algorithm combines a nested iteration with multigrid cycling, with approximations of coarser grids used as initial guesses for multigrid cycles on finer grids of the hierarchy. More specifically, this algorithm starts from the coarsest grid, $\Omega_{\ell}$, solving the corresponding discrete problem to obtain a solution, $u_{\ell}^{F M G}$. This solution is interpolated to the next finer grid, obtaining an initial guess, $u_{\ell-1}^{0}=J_{\ell}^{\ell-1} u_{\ell}^{F M G}$, where $J_{\ell}^{\ell-1}: \mathcal{G}\left(\Omega_{\ell}\right) \rightarrow \mathcal{G}\left(\Omega_{\ell-1}\right)$ is a suitable prolongation operator. Starting from this initial approximation, $\nu$ cycles of a suitable multigrid algorithm are performed to obtain the approximation $u_{\ell-1}^{F M G}$ on grid $\Omega_{\ell-1}$. This solution is interpolated to the next finer grid, by means of a prolongation operator $J_{k+1}^{k}: \mathcal{G}\left(\Omega_{k+1}\right) \rightarrow \mathcal{G}\left(\Omega_{k}\right)$, followed again by $\nu$ cycles, and so forth, until the target grid is reached. On the finest grid, again (typically) $\nu$ cycles are performed, yielding the final solution, $u_{1}^{F M G}$. The computation of $u_{k}^{F M G}$ from $u_{k+1}^{F M G}$ is illustrated in Figure 2.1.

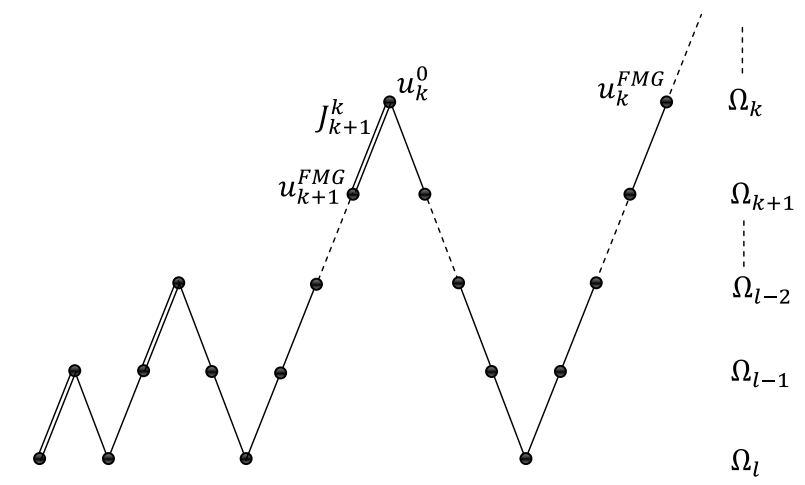

FIG. 2.1. Computation of $u_{k}^{F M G}$ from $u_{k+1}^{F M G}$ in the case $\nu=1$.

The aim of the FMG method is to achieve discretization-level accuracy with just one or a few iterations of an appropriate multigrid algorithm at each level of the hierarchy. The purpose in this work is to obtain a quantitative bound for the accuracy of the approximation obtained by FMG. Our goal is to provide an FMG algorithm which yields a total error that is comparable to the discretizaton error:

$$
\left\|\mathcal{J}_{1} u-u_{1}^{F M G}\right\| \approx\left\|\mathcal{J}_{1} u-u_{1}\right\|
$$

where $u$ is the solution of the continuous problem, $\mathcal{J}_{1}$ is the operator that transfers continuous functions to the discrete grid function space, and $\|\cdot\|$ is some appropriate norm. By definition, for any grid $\Omega_{k}$ in the hierarchy,

$$
u_{k}-u_{k}^{F M G}=\left(M_{k}^{\ell-k+1}\right)^{\nu}\left(u_{k}-u_{k}^{0}\right),
$$

where $M_{k}^{\ell-k+1}$ is the iteration matrix on grid level $k$ corresponding to the multigrid cycle that employs $\ell-k+1$ grid levels. Following the steps of the algorithm (see [15]), one obtains the following error propagation matrix for the multigrid cycle,

$$
\begin{aligned}
M_{k}^{\ell-k+1} & =S_{k}^{\nu_{2}}\left(I_{k}-I_{k+1}^{k}\left(I_{k+1}-\left(M_{k+1}^{\ell-k}\right)^{\nu}\right) L_{k+1}^{-1} I_{k}^{k+1} L_{k}\right) S_{k}^{\nu_{1}}, k=1, \ldots, \ell-1, \\
M_{\ell}^{1} & =0 .
\end{aligned}
$$


Here, $S_{k}$ is some smoothing (relaxation) operator, $\nu_{1}$ and $\nu_{2}$ are the numbers of pre- and post-smoothing steps, $I_{k}$ is the identity operator belonging to $\mathcal{G}\left(\Omega_{k}\right)$, and $I_{k+1}^{k}: \mathcal{G}\left(\Omega_{k+1}\right) \rightarrow \mathcal{G}\left(\Omega_{k}\right)$, and $I_{k}^{k+1}: \mathcal{G}\left(\Omega_{k}\right) \rightarrow \mathcal{G}\left(\Omega_{k+1}\right)$, are the prolongation and restriction operators, respectively.

On the other hand, the initial algebraic $k$-level error, $u_{k}-u_{k}^{0}$, can be expressed by applying $G_{k}^{\ell-k+1}$ to the right-hand side $f_{k}$ :

$$
u_{k}-u_{k}^{0}=G_{k}^{\ell-k+1} f_{k},
$$

with $G_{k}^{\ell-k+1}: \mathcal{G}\left(\Omega_{k}\right) \rightarrow \mathcal{G}\left(\Omega_{k}\right)$. Now, following the steps of the FMG algorithm we find that

$$
\begin{aligned}
G_{k}^{\ell-k+1} & =L_{k}^{-1}-J_{k+1}^{k}\left(L_{k+1}^{-1}-\left(M_{k+1}^{\ell-k}\right)^{\nu} G_{k+1}^{\ell-k}\right) J_{k}^{k+1}, k=1, \ldots, \ell-1, \\
G_{\ell}^{1} & =0,
\end{aligned}
$$

where $M_{k+1}^{\ell-k}$ is the multigrid iteration operator on level $k+1$ using $\ell-k$ levels, as previously defined. The restriction and prolongation operators, $J_{k}^{k+1}$ and $J_{k+1}^{k}$, respectively, may be different from those used in the multigrid cycle $M_{k}^{\ell-k+1}$.

In the particular case where only two levels are used, the structure of the FMG iteration operator is depicted in Figure 2.2. In this case, the initial algebraic error on the finest level is given by

$$
u_{1}-u_{1}^{0}=G_{1}^{2} \mathcal{J}_{1} f=\left(L_{1}^{-1}-J_{2}^{1} L_{2}^{-1} J_{1}^{2}\right) \mathcal{J}_{1} f,
$$

and the algebraic error yielded by the FMG algorithm is

$$
u_{1}-u_{1}^{F M G}=\left(M_{1}^{2}\right)^{\nu} G_{1}^{2} \mathcal{J}_{1} f=\left(M_{1}^{2}\right)^{\nu}\left(L_{1}^{-1}-J_{2}^{1} L_{2}^{-1} J_{1}^{2}\right) \mathcal{J}_{1} f,
$$

with $M_{1}^{2}$ the usual two-level operator given by

$$
M_{1}^{2}=S_{1}^{\nu_{2}}\left(I_{1}-I_{2}^{1} L_{2}^{-1} I_{1}^{2} L_{1}\right) S_{1}^{\nu_{1}} .
$$

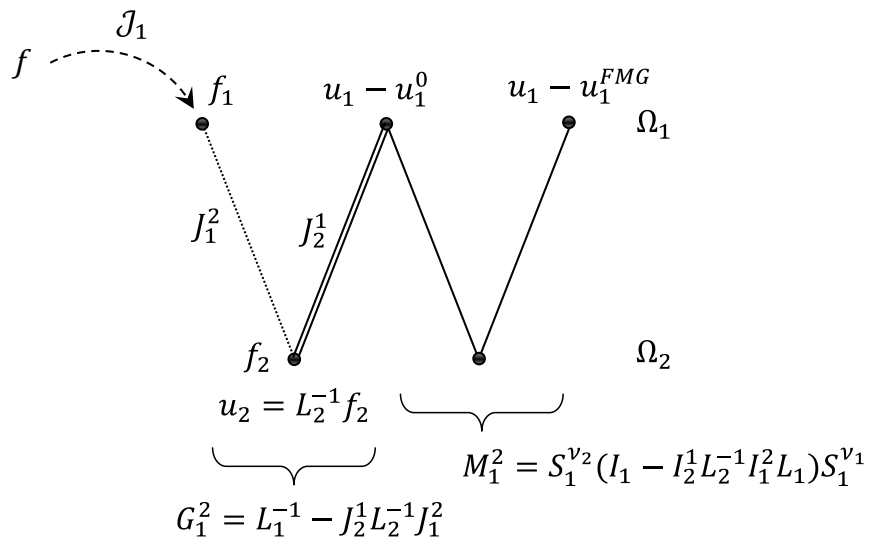

FIG. 2.2. The FMG two-level iteration operator.

The discretization error on the finest grid, $\mathcal{J}_{1} u-u_{1}$, can also be written as an application of the operator $\mathcal{T}_{1}: L^{2}\left(\mathbb{R}^{d}\right) \rightarrow \mathcal{G}\left(\Omega_{1}\right)$ to the continuous right-hand side $f$; that is,

$$
\mathcal{J}_{1} u-u_{1}=\mathcal{T}_{1} f=\left(\mathcal{J}_{1} \mathcal{L}^{-1}-L_{1}^{-1} \mathcal{J}_{1}\right) f .
$$


Thus, we can rewrite the total error, $\mathcal{J}_{1} u-u_{1}^{F M G}$, as follows:

$$
\mathcal{J}_{1} u-u_{1}^{F M G}=\left(\mathcal{J}_{1} u-u_{1}\right)+\left(u_{1}-u_{1}^{F M G}\right)=\mathcal{F}_{1}^{\ell} f
$$

by defining the operator $\mathcal{F}_{1}^{\ell}: L^{2}\left(\mathbb{R}^{d}\right) \rightarrow \mathcal{G}\left(\Omega_{1}\right)$ as

$$
\mathcal{F}_{1}^{\ell}=\mathcal{T}_{1}+\left(M_{1}^{\ell}\right)^{\nu} G_{1}^{\ell} \mathcal{J}_{1}
$$

To compare the total error corresponding to the solution obtained by FMG with the discretization error, the following worst-case definition is introduced.

DeFINITION 2.1. The FMG accuracy measure using $\ell$ grid levels, denoted by $E_{F M G}^{\ell}$, is defined as

$$
E_{F M G}^{\ell}=\sup _{f \in \mathcal{E}}\left\{\frac{\left\|\mathcal{F}_{1}^{\ell} f\right\|}{\left\|\mathcal{T}_{1} f\right\|}\right\}
$$

where $\mathcal{E}$ is a set of allowable right-hand sides, and $\mathcal{T}_{1}$ and $\mathcal{F}_{1}^{\ell}$ are defined in (2.5) and (2.6), respectively.

Remark. The set of allowable $f$ 's, denoted by $\mathcal{E}$, needs to be selected with care, so as to avoid cases where the discretization error "accidentally vanishes" on the grid (or nearly so). This can happen, in particular, when $f$ contains modes that are unresolved on the finest grid. We return to this point in the next section, but next an explicit example illustrating such trouble is presented.

Example 2.1. Let us consider the one-dimensional Poisson differential equation,

$$
L u=-\partial_{x x} u=f,
$$

discretized on a grid with mesh-size $h_{k}$ as follows:

$$
L_{k} u_{k}=-\beta\left(\partial_{x x}\right)_{k} u_{k}-(1-\beta)\left(\partial_{x x}\right)_{k+1} u_{k},
$$

where $\beta$ is a constant, and the discrete operators $\left(\partial_{x x}\right)_{k}$ and $\left(\partial_{x x}\right)_{k+1}$ are the standard three-point finite difference discretizations for the second derivative with mesh-sizes $h_{k}$ and $h_{k+1}$, respectively.

If we fix the mesh-size $h_{k}$ and some single Fourier frequency $\theta \in(-\pi, \pi]$, it is possible to find a discretization, i.e., a constant $\beta$, for which the discretization error vanishes, because the symbol of the discrete operator equals that of the differential operator. In fact, if Fourier symbols (cf. section 3) of the continuum operator and the discrete one are

$$
\begin{aligned}
\widetilde{L}(\theta) & =\frac{\theta^{2}}{h_{k}^{2}}, \\
\widetilde{L_{k}}(\theta) & =\beta \frac{4}{h_{k}^{2}} \sin ^{2}\left(\frac{\theta}{2}\right)+(1-\beta) \frac{1}{h_{k}^{2}} \sin ^{2}(\theta),
\end{aligned}
$$

equating both and solving for $\beta$, we get the following value:

$$
\beta=\frac{\theta^{2}-\sin ^{2}(\theta)}{4 \sin ^{2}\left(\frac{\theta}{2}\right)-\sin ^{2}(\theta)} .
$$

Thus, for any frequency $\theta$, it is possible to find a value of $\beta$ for which "accidental vanishing" of the discretization error occurs. For example for $\theta=\pi$ a value of $\beta=\pi^{2} / 4$ gives a discretization with this behavior independently of the grid. 
3. Local Fourier analysis for FMG. In this section, we introduce the local Fourier analysis framework to estimate the FMG accuracy measure for $k$ levels, $E_{F M G}^{k}$, given in Definition 2.1. We use $k$ to denote the number of levels used in the analysis, as opposed to $\ell$ - the number of levels used in actual numerical application of the FMG algorithm. In section 3.1 we present the basic principles and some notation required for the $k$-level Fourier analysis. To keep the presentation as simple as possible, we restrict ourselves to the two-dimensional scalar case and to standard coarsening. The generalization to higher dimensions or to systems of equations is easily done with the usual modifications, making the analysis more technically involved. Section 3.2 is devoted to the two-level FMG Fourier analysis, and some results are presented to confirm the theoretical estimates obtained by this analysis. Finally, in section 3.3 the analysis is extended to the case of $k$ levels, and some numerical results validating the predictions are provided.

3.1. Basic principles of local Fourier analysis. The main idea in the local Fourier analysis is formally to extend all multigrid components to an infinite grid, neglecting the boundary conditions, and to restrict the analysis to discrete linear operators with constant coefficients. Therefore, for a $k$-level cycle, we consider $k$ discrete linear operators $L_{j}, j=1, \ldots, k$ on $k$ infinite grids $G_{j}$, with mesh sizes $h_{j}=2^{j-1} h, j=1, \ldots, k$,

$$
G_{j}=\left\{\mathbf{x}=\left(x_{1}, x_{2}\right) \mid x_{i}=k_{i} h_{j}, k_{i} \in \mathbb{Z}, i=1,2\right\} .
$$

For a fixed grid point $\mathrm{x} \in G_{j}$ the corresponding equation of the discrete problem $L_{j} u_{j}=f_{j}$ extended to the infinite grid, $G_{j}$, reads in stencil notation [14],

$$
L_{j} u_{j}(\mathbf{x})=\sum_{\kappa=\left(\kappa_{1}, \kappa_{2}\right) \in \mathcal{I}_{j}} s_{\kappa}^{j} u_{j}\left(x_{1}+\kappa_{1} h_{j}, x_{2}+\kappa_{2} h_{j}\right)=f_{j}(\mathbf{x}),
$$

where $s_{\kappa}^{j} \in \mathbb{R}$ are constant coefficients, and $\mathcal{I}_{j} \subset \mathbb{Z}^{2}$ are finite index sets. From (3.2), it can be deduced that the grid functions $\varphi_{j}(\boldsymbol{\theta}, \mathbf{x})=e^{i \boldsymbol{\theta} \mathbf{x}}=e^{i \theta_{1} x_{1}} e^{i \theta_{2} x_{2}}$, where the Fourier frequencies $\boldsymbol{\theta}=\left(\theta_{1}, \theta_{2}\right)$ vary continuously in $\mathbb{R}^{2}$, are formal eigenfunctions of the discrete operator $L_{j}$. More precisely, the relation $L_{j} \varphi_{j}(\boldsymbol{\theta}, \mathbf{x})=\widetilde{L}_{j}(\boldsymbol{\theta}) \varphi_{j}(\boldsymbol{\theta}, \mathbf{x})$ holds, where

$$
\widetilde{L}_{j}(\boldsymbol{\theta})=\sum_{\boldsymbol{\kappa} \in \mathcal{I}_{j}} s_{\kappa}^{j} e^{i\left(\theta_{1} \kappa_{1}+\theta_{2} \kappa_{2}\right) h_{j}}
$$

is the corresponding eigenvalue or Fourier symbol of $L_{j}$.

For $u_{j}, v_{j}$, discrete grid functions defined on $G_{j}$, we consider the following discrete inner product:

$$
<u_{j}, v_{j}>=h_{j}^{2} \sum_{\mathbf{x} \in G_{j}} u_{j}(\mathbf{x}) \overline{v_{j}(\mathbf{x})},
$$

and we introduce the space of bounded infinite grid functions,

$$
l_{j}^{2}\left(G_{j}\right)=\left\{u_{j}: G_{j} \rightarrow \mathbb{C}|| u_{j} \|<\infty\right\},
$$

where $\|\cdot\|$ is the norm associated with the inner product (3.4). From the inverse Fourier transformation, each function $u_{j} \in l_{j}^{2}\left(G_{j}\right)$ can be written as a formal linear combination of the Fourier modes, $\varphi_{j}(\boldsymbol{\theta}, \mathbf{x})$; see $[4,7,13]$. Due to the periodicity of these 
functions, those associated with Fourier frequencies satisfying $\max \left\{\left|\theta_{1}\right|,\left|\theta_{2}\right|\right\} \geq \pi / h_{j}$ coincide with certain Fourier modes with $\boldsymbol{\theta} \in \Theta_{j}=\left(-\pi / h_{j}, \pi / h_{j}\right]^{2}$, and therefore, the Fourier space,

$$
\mathcal{E}_{j}=\operatorname{span}\left\{\varphi_{j}(\boldsymbol{\theta}, \cdot) \mid \boldsymbol{\theta} \in \Theta_{j}\right\},
$$

generates any bounded infinite grid function on $G_{j}[4]$. We would also like to remark that the term span means the formal linear combination, as referred to before. In particular, the discrete solution on the finest level, $u_{1}$, and the initial approximation, $u_{1}^{0}$, can be represented as linear combinations of grid functions in the Fourier space, and therefore this applies also to the initial algebraic error, $e_{1}^{0}=u_{1}-u_{1}^{0}$. The same holds for the error obtained after the application of $\nu$ iterations of a $k$-level cycle, $e_{1}^{\nu}=u_{1}-u_{1}^{\nu}=\left(M_{1}^{k}\right)^{\nu} e_{1}^{0}$, with $M_{1}^{k}$ as in (2.3). For all the operators involved in the recursive definition of $M_{1}^{k}$, a stencil representation can be formulated, and therefore the corresponding Fourier symbols can be computed similarly to (3.3) for the discrete operators $L_{j}$. In $[14,15,16,18]$, the symbols for a wide range of restriction, prolongation, and discrete operators are tabulated.

3.2. Two-level FMG analysis. The goal in this section is to use the local Fourier analysis described in section 3.1 to estimate the FMG accuracy measure given in Definition 2.1 for the two-level case, $E_{F M G}^{2}$. Assuming an infinite grid and standard coarsening, the Fourier space $\mathcal{E}_{1}$ is decomposed into the following four-dimensional subspaces, known as $2 h$-harmonics,

$$
\mathcal{E}_{1}^{4}\left(\boldsymbol{\theta}^{00}\right)=\operatorname{span}\left\{\varphi_{1}\left(\boldsymbol{\theta}^{00}, \cdot\right), \varphi_{1}\left(\boldsymbol{\theta}^{11}, \cdot\right), \varphi_{1}\left(\boldsymbol{\theta}^{10}, \cdot\right), \varphi_{1}\left(\boldsymbol{\theta}^{01}, \cdot\right)\right\}, \quad \boldsymbol{\theta}^{00} \in \Theta_{2},
$$

where

$$
\begin{aligned}
& \boldsymbol{\theta}^{10}=\boldsymbol{\theta}^{00}-\left(\operatorname{sign}\left(\theta_{1}^{00}\right) \pi / h, 0\right), \\
& \boldsymbol{\theta}^{01}=\boldsymbol{\theta}^{00}-\left(0, \operatorname{sign}\left(\theta_{2}^{00}\right) \pi / h\right), \\
& \boldsymbol{\theta}^{11}=\boldsymbol{\theta}^{00}-\left(\operatorname{sign}\left(\theta_{1}^{00}\right) \pi / h, \operatorname{sign}\left(\theta_{2}^{00}\right) \pi / h\right) .
\end{aligned}
$$

Namely, each grid function associated with a low frequency $\boldsymbol{\theta}^{00} \in \Theta_{2}$ is coupled with three grid functions associated with high frequencies $\boldsymbol{\theta}^{10}, \boldsymbol{\theta}^{01}, \boldsymbol{\theta}^{11} \in \Theta_{1}$, in the sense that these three high frequencies coincide on the coarse grid $G_{2}$ with the low frequency. With these definitions, many common smoothers $S_{1}$ are invariant with respect to the set of $2 h$-harmonics, $S_{1}: \mathcal{E}_{1}^{4}\left(\boldsymbol{\theta}^{00}\right) \rightarrow \mathcal{E}_{1}^{4}\left(\boldsymbol{\theta}^{00}\right)$, and this then also applies to the two-level operator $M_{1}^{2}$ for an arbitrary Fourier frequency $\boldsymbol{\theta}^{00} \in \widetilde{\Theta}_{2}=\Theta_{2} \backslash \Psi_{2}$, where

$$
\Psi_{2}=\left\{\boldsymbol{\theta}^{00} \in \Theta_{2} \mid \widetilde{L}_{2}\left(2 \boldsymbol{\theta}^{00}\right)=0, \text { or } \widetilde{L}_{1}\left(\boldsymbol{\theta}^{i j}\right)=0, i j \in\{00,11,10,01\}\right\} ;
$$

i.e., $M_{1}^{2}: \mathcal{E}_{1}^{4}\left(\boldsymbol{\theta}^{00}\right) \rightarrow \mathcal{E}_{1}^{4}\left(\boldsymbol{\theta}^{00}\right)$, with $\boldsymbol{\theta}^{00} \in \widetilde{\Theta}_{2}$. Note that $\left.M_{1}^{2}\right|_{\mathcal{E}_{1}^{4}\left(\boldsymbol{\theta}^{00}\right)}$ can be represented by a $4 \times 4$ eigenmatrix, ${ }^{1}$ denoted here by $M_{1}^{2 \ell}\left(\boldsymbol{\theta}^{00}\right)$. With respect to the operator $G_{1}^{2}=$ $L_{1}^{-1}-J_{2}^{1} L_{2}^{-1} J_{1}^{2}$, the same invariance property is fulfilled; i.e., $G_{1}^{2}: \mathcal{E}_{1}^{4}\left(\boldsymbol{\theta}^{00}\right) \rightarrow \mathcal{E}_{1}^{4}\left(\boldsymbol{\theta}^{00}\right)$, for each $\boldsymbol{\theta}^{00} \in \widetilde{\Theta}_{2}$, and it holds that

$$
G_{1}^{2 \ell}\left(\boldsymbol{\theta}^{00}\right):=\left.G_{1}^{2}\right|_{\mathcal{E}_{1}^{4}\left(\boldsymbol{\theta}^{00}\right)}=\left(L_{1}^{2 \ell}\left(\boldsymbol{\theta}^{00}\right)\right)^{-1}-\left(J_{2}^{1}\right)^{2 \ell}\left(\boldsymbol{\theta}^{00}\right)\left(L_{2}^{2 \ell}\left(\boldsymbol{\theta}^{00}\right)\right)^{-1}\left(J_{1}^{2}\right)^{2 \ell}\left(\boldsymbol{\theta}^{00}\right),
$$

\footnotetext{
${ }^{1}$ Here, the term eigenmatrix generalizes the classical term eigenvalue in the sense that it is a block associated with an invariant subspace. Thus, an eigenvalue is an eigenmatrix of size 1, and its eigenvector corresponds to the invariant subspace.
} 
where

$$
\begin{aligned}
L_{1}^{2 \ell}\left(\boldsymbol{\theta}^{00}\right) & =\operatorname{diag}\left\{\widetilde{L_{1}}\left(\boldsymbol{\theta}^{00}\right), \widetilde{L_{1}}\left(\boldsymbol{\theta}^{11}\right), \widetilde{L_{1}}\left(\boldsymbol{\theta}^{10}\right), \widetilde{L_{1}}\left(\boldsymbol{\theta}^{01}\right)\right\} \in \mathbb{C}^{4 \times 4}, \\
\left(J_{2}^{1}\right)^{2 \ell}\left(\boldsymbol{\theta}^{00}\right) & =\left(\widetilde{J_{2}^{1}}\left(\boldsymbol{\theta}^{00}\right), \widetilde{J_{2}^{1}}\left(\boldsymbol{\theta}^{11}\right), \widetilde{J_{2}^{1}}\left(\boldsymbol{\theta}^{10}\right), \widetilde{J_{2}^{1}}\left(\boldsymbol{\theta}^{01}\right)\right)^{t} \in \mathbb{C}^{4 \times 1}, \\
\left(J_{1}^{2}\right)^{2 \ell}\left(\boldsymbol{\theta}^{00}\right) & =\left(\widetilde{J_{1}^{2}}\left(\boldsymbol{\theta}^{00}\right), \widetilde{J_{1}^{2}}\left(\boldsymbol{\theta}^{11}\right), \widetilde{J_{1}^{2}}\left(\boldsymbol{\theta}^{10}\right), \widetilde{J_{1}^{2}}\left(\boldsymbol{\theta}^{01}\right)\right) \in \mathbb{C}^{1 \times 4}, \\
L_{2}^{2 \ell}\left(\boldsymbol{\theta}^{00}\right) & =\widetilde{L_{2}}\left(2 \boldsymbol{\theta}^{00}\right) \in \mathbb{C}^{1 \times 1} .
\end{aligned}
$$

Next, we consider the remaining operators appearing in Definition 2.1 of $E_{F M G}^{2}$, in particular, $\mathcal{T}_{1}$, and $\mathcal{J}_{1}$. These operators map continuous functions to discrete functions. In the proposed FMG analysis we restrict ourselves to the following set of continuous functions:

$$
\mathcal{E}=\operatorname{span}\left\{\varphi(\boldsymbol{\theta}, \mathbf{x})=e^{i \boldsymbol{\theta} \mathbf{x}} \mid \mathbf{x} \in \mathbb{R}^{2}, \boldsymbol{\theta} \in \Theta_{1}\right\} .
$$

The restriction $f \in \mathcal{E}$ ensures that $f$ is sufficiently smooth to be sampled on the fine grid without any aliasing. This restriction can be viewed in the general context of the restriction of Fourier transforms [12], which is very useful in practice for the multidimensional case. Without this restriction, it is possible to choose a combination of frequencies (that alias with each other on the finest grid), for which the discretization error vanishes on the grid, rendering our definition useless; see Example 2.1.

For each $\boldsymbol{\theta}^{00} \in \Theta_{2}$, we define the four-dimensional subspace of $\mathcal{E}$ by

$$
\mathcal{E}^{4}\left(\boldsymbol{\theta}^{00}\right)=\operatorname{span}\left\{\varphi\left(\boldsymbol{\theta}^{00}, \cdot\right), \varphi\left(\boldsymbol{\theta}^{11}, \cdot\right), \varphi\left(\boldsymbol{\theta}^{10}, \cdot\right), \varphi\left(\boldsymbol{\theta}^{01}, \cdot\right)\right\}
$$

where again $\boldsymbol{\theta}^{00}, \boldsymbol{\theta}^{11}, \boldsymbol{\theta}^{10}$, and $\boldsymbol{\theta}^{01}$ are defined as in (3.7). As $\mathcal{J}_{1} \varphi(\boldsymbol{\theta}, \mathbf{x})=$ $\varphi_{1}(\boldsymbol{\theta}, \mathbf{x}), \forall \mathbf{x} \in G_{1}$, it is fulfilled that $\mathcal{T}_{1}: \mathcal{E}^{4}\left(\boldsymbol{\theta}^{00}\right) \rightarrow \mathcal{E}_{1}^{4}\left(\boldsymbol{\theta}^{00}\right)$. In fact, from the definition $\mathcal{T}_{1}=\mathcal{J}_{1} \mathcal{L}^{-1}-L_{1}^{-1} \mathcal{J}_{1}$, we find that

$$
\mathcal{T}_{1} \varphi\left(\boldsymbol{\theta}^{i j}, \mathbf{x}\right)=\left(\widetilde{\mathcal{L}}^{-1}\left(\boldsymbol{\theta}^{i j}\right)-\widetilde{L}_{1}^{-1}\left(\boldsymbol{\theta}^{i j}\right)\right) \varphi_{1}\left(\boldsymbol{\theta}^{i j}, \mathbf{x}\right) \forall \mathbf{x} \in G_{1} \text {, with } i j \in\{00,11,10,01\},
$$

where $\widetilde{\mathcal{L}}\left(\boldsymbol{\theta}^{i j}\right)$ is the Fourier symbol of the continuous operator $\mathcal{L}$; i.e., $\mathcal{L} \varphi\left(\boldsymbol{\theta}^{i j}, \mathbf{x}\right)=$ $\widetilde{\mathcal{L}}\left(\boldsymbol{\theta}^{i j}\right) \varphi\left(\boldsymbol{\theta}^{i j}, \mathbf{x}\right)$. We will call $\widetilde{\mathcal{T}}_{1}\left(\boldsymbol{\theta}^{i j}\right)=\widetilde{\mathcal{L}}^{-1}\left(\boldsymbol{\theta}^{i j}\right)-\widetilde{L}_{1}^{-1}\left(\boldsymbol{\theta}^{i j}\right)$ the Fourier symbol of operator $\mathcal{T}_{1}$. Let us consider an arbitrary $f \in \mathcal{E}^{4}\left(\boldsymbol{\theta}^{00}\right)$, with coordinates $\mathbf{v}=$ $\left(\alpha_{00}, \alpha_{11}, \alpha_{10}, \alpha_{01}\right)^{t}$ with respect to the basis $\left\{\varphi\left(\boldsymbol{\theta}^{00}, \cdot\right), \varphi\left(\boldsymbol{\theta}^{11}, \cdot\right), \varphi\left(\boldsymbol{\theta}^{10}, \cdot\right), \varphi\left(\boldsymbol{\theta}^{01}, \cdot\right)\right\}$,

$$
f(\mathbf{x})=\alpha_{00} \varphi\left(\boldsymbol{\theta}^{00}, \mathbf{x}\right)+\alpha_{11} \varphi\left(\boldsymbol{\theta}^{11}, \mathbf{x}\right)+\alpha_{10} \varphi\left(\boldsymbol{\theta}^{10}, \mathbf{x}\right)+\alpha_{01} \varphi\left(\boldsymbol{\theta}^{01}, \mathbf{x}\right), \mathbf{x} \in \mathbb{R}^{2} .
$$

Then, we have $\mathcal{T}_{1} f(\mathbf{x})=\beta_{00} \varphi_{1}\left(\boldsymbol{\theta}^{00}, \mathbf{x}\right)+\beta_{11} \varphi_{1}\left(\boldsymbol{\theta}^{11}, \mathbf{x}\right)+\beta_{10} \varphi_{1}\left(\boldsymbol{\theta}^{10}, \mathbf{x}\right)+\beta_{01} \varphi_{1}\left(\boldsymbol{\theta}^{01}, \mathbf{x}\right)$, where the coefficients $\beta_{i j}$ are related to the coefficients $\alpha_{i j}$ by the following expression:

$$
\left(\begin{array}{c}
\beta_{00} \\
\beta_{11} \\
\beta_{10} \\
\beta_{01}
\end{array}\right)=\left(\begin{array}{cccc}
\widetilde{\mathcal{T}}_{1}\left(\boldsymbol{\theta}^{00}\right) & 0 & 0 & 0 \\
0 & \widetilde{\mathcal{T}}_{1}\left(\boldsymbol{\theta}^{11}\right) & 0 & 0 \\
0 & 0 & \widetilde{\mathcal{T}}_{1}\left(\boldsymbol{\theta}^{10}\right) & 0 \\
0 & 0 & 0 & \widetilde{\mathcal{T}}_{1}\left(\boldsymbol{\theta}^{01}\right)
\end{array}\right)\left(\begin{array}{c}
\alpha_{00} \\
\alpha_{11} \\
\alpha_{10} \\
\alpha_{01}
\end{array}\right)=\mathcal{T}_{1}^{2 \ell}\left(\boldsymbol{\theta}^{00}\right)\left(\begin{array}{c}
\alpha_{00} \\
\alpha_{11} \\
\alpha_{10} \\
\alpha_{01}
\end{array}\right) .
$$

Since $\mathcal{T}_{1}: \mathcal{E}^{4}\left(\boldsymbol{\theta}^{00}\right) \rightarrow \mathcal{E}_{1}^{4}\left(\boldsymbol{\theta}^{00}\right)$ and $\left(M_{1}^{2}\right)^{\nu} G_{1}^{2} \mathcal{J}_{1}: \mathcal{E}^{4}\left(\boldsymbol{\theta}^{00}\right) \rightarrow \mathcal{E}_{1}^{4}\left(\boldsymbol{\theta}^{00}\right)$, it is straightforward to see that also $\mathcal{F}_{1}^{2}: \mathcal{E}^{4}\left(\boldsymbol{\theta}^{00}\right) \rightarrow \mathcal{E}_{1}^{4}\left(\boldsymbol{\theta}^{00}\right)$, with

$$
\mathcal{F}_{1}^{2 \ell}\left(\boldsymbol{\theta}^{00}\right):=\mathcal{T}_{1}^{2 \ell}\left(\boldsymbol{\theta}^{00}\right)+\left(M_{1}^{2 \ell}\left(\boldsymbol{\theta}^{00}\right)\right)^{\nu} G_{1}^{2 \ell}\left(\boldsymbol{\theta}^{00}\right)
$$

the $4 \times 4$ eigenmatrix representation of $\mathcal{F}_{1}^{2}$ with respect to the subspaces $\mathcal{E}^{4}\left(\boldsymbol{\theta}^{00}\right)$ and $\mathcal{E}_{1}^{4}\left(\boldsymbol{\theta}^{00}\right)$. 
From our assumptions on the right-hand side $f$, using the representation of $\mathcal{F}_{1}^{2}$ by the $4 \times 4$ eigenmatrix $\mathcal{F}_{1}^{2 \ell}\left(\boldsymbol{\theta}^{00}\right)$, we can estimate the two-level FMG accuracy measure by

$$
\widetilde{E}_{F M G}^{2}=\sup _{\boldsymbol{\theta}^{00} \in \widetilde{\Theta}_{2}}\left\{\sup _{\substack{\mathbf{v} \in \mathbb{C} \\ \mathbf{v} \neq \mathbf{0}}} \frac{\left\|\mathcal{F}_{1}^{2 \ell}\left(\boldsymbol{\theta}^{00}\right) \mathbf{v}\right\|}{\left\|\mathcal{T}_{1}^{2 \ell}\left(\boldsymbol{\theta}^{00}\right) \mathbf{v}\right\|} ; \operatorname{det}\left(\mathcal{T}_{1}^{2 \ell}\left(\boldsymbol{\theta}^{00}\right)\right) \neq 0\right\} .
$$

Using

$$
\left\|\mathcal{F}_{1}^{2 \ell}\left(\boldsymbol{\theta}^{00}\right)\left(\mathcal{T}_{1}^{2 \ell}\left(\boldsymbol{\theta}^{00}\right)\right)^{-1}\right\|=\sup _{\substack{\mathbf{w} \in \mathbb{C} \\ \mathbf{w} \neq \mathbf{0}}} \frac{\left\|\mathcal{F}_{1}^{2 \ell}\left(\boldsymbol{\theta}^{00}\right)\left(\mathcal{T}_{1}^{2 \ell}\left(\boldsymbol{\theta}^{00}\right)\right)^{-1} \mathbf{w}\right\|}{\|\mathbf{w}\|}=\sup _{\substack{\mathbf{v} \in \mathbb{C}^{4}, \mathbf{v} \neq 0}} \frac{\left\|\mathcal{F}_{1}^{2 \ell}\left(\boldsymbol{\theta}^{00}\right) \mathbf{v}\right\|}{\left\|\mathcal{T}_{1}^{2 \ell}\left(\boldsymbol{\theta}^{00}\right) \mathbf{v}\right\|},
$$

we can simplify the expression for $\widetilde{E}_{F M G}^{2}$ as follows:

$$
\widetilde{E}_{F M G}^{2}=\sup _{\boldsymbol{\theta}^{00} \in \widetilde{\Theta}_{2}}\left\{\left\|\mathcal{F}_{1}^{2 \ell}\left(\boldsymbol{\theta}^{00}\right)\left(\mathcal{T}_{1}^{2 \ell}\left(\boldsymbol{\theta}^{00}\right)\right)^{-1}\right\| ; \operatorname{det}\left(\mathcal{T}_{1}^{2 \ell}\left(\boldsymbol{\theta}^{00}\right)\right) \neq 0\right\} .
$$

The estimation of the two-level FMG accuracy measure, $\widetilde{E}_{F M G}^{2}$, is thus reduced to calculating norms of $4 \times 4$ matrices. As the Fourier space (3.5) has a non-denumerable basis, $\boldsymbol{\theta}$ varies continuously in $\Theta_{1}$. In general, the suprema from (3.12) and (3.14) cannot be calculated analytically, so the practical computations in the next section are restricted to a finite-dimensional Fourier space.

3.2.1. Validation of the two-level FMG analysis. In order to validate the estimate of the accuracy measure, $\widetilde{E}_{F M G}^{2}$, defined in (3.14), we introduce the concept of the worst-case right-hand side analysis. By means of the two-level FMG analysis proposed above, we obtain a value for $\widetilde{E}_{F M G}^{2}$, which is realized for a specific $\boldsymbol{\theta}^{00}=$ $\left(\theta_{1}^{00}, \theta_{2}^{00}\right) \in \widetilde{\Theta}_{2}$. We expect that the difference between the computed solution on the fine grid and the exact solution of the continuous problem, is at most $\left(\widetilde{E}_{F M G}^{2}\right)$ times the fine grid discretization error. This ratio, given in (3.12), is attained for some vectors $\mathbf{v}=\left(\alpha_{00}, \alpha_{11}, \alpha_{10}, \alpha_{01}\right)^{t} \in \mathbb{C}^{4}$, which define specific right-hand sides $f$, as in (3.11). When the two-norm matrix is used, from (3.13) a vector $\mathbf{v}$ can be found by the expression $\mathbf{v}=\left(\mathcal{T}_{1}^{2 \ell}\left(\boldsymbol{\theta}^{00}\right)\right)^{-1} \mathbf{w}$, with $\mathbf{w}$ an eigenvector of the $4 \times 4$ matrix $W\left(\boldsymbol{\theta}^{00}\right)^{t} W\left(\boldsymbol{\theta}^{00}\right)$, associated with its maximum modulus eigenvalue, where $W\left(\boldsymbol{\theta}^{00}\right)=$ $\mathcal{F}_{1}^{2 \ell}\left(\boldsymbol{\theta}^{00}\right)\left(\mathcal{T}_{1}^{2 \ell}\left(\boldsymbol{\theta}^{00}\right)\right)^{-1}$. It is thus possible to find the right-hand side for which the ratio given by the analysis is obtained; that is, one can find the worst-case two-level LFA convergence in practice. This permits us to validate the two-level FMG analysis by employing this specific right-hand side.

To illustrate the process, we use the Poisson equation in a unit square domain as our model problem, with periodic boundary conditions,

$$
\begin{aligned}
\mathcal{L} u(x, y) & =-\Delta u(x, y)=f(x, y), \quad(x, y) \in(0,1) \times(0,1), \\
u(x, 0) & =u(x, 1), \quad x \in[0,1], \\
u(0, y) & =u(1, y), \quad y \in[0,1] .
\end{aligned}
$$

The standard five-point discretization on the fine and coarse grids, $L_{k} u_{k}=f_{k}, k=$ 1,2 , is considered. We use injection for $J_{1}^{2}$, bilinear interpolation for both $J_{2}^{1}$, and $I_{2}^{1}$, and full-weighting restriction for $I_{1}^{2}$. The smoother (relaxation), is damped Jacobi with parameter $\omega=0.8$, applying one pre- and one post-smoothing step. A single twolevel cycle, i.e., $\nu=1$ with $\nu$ as in (2.2), is performed in this experiment. Employing a 
grid of size $64 \times 64$ for the frequency space, the ratio predicted by the two-level FMG analysis is $\widetilde{E}_{F M G}^{2}=6.13$ for this example. For each low frequency $\boldsymbol{\theta}^{00}$ which satisfies $\operatorname{det}\left(\mathcal{T}_{1}^{2 \ell}\left(\boldsymbol{\theta}^{00}\right)\right) \neq 0$, the factor

$$
\widetilde{E}_{F M G}^{2}\left(\boldsymbol{\theta}^{00}\right)=\sup _{\substack{\mathbf{v} \in \mathbb{C}^{4} \\ \mathbf{v} \neq \mathbf{0}}} \frac{\left\|\mathcal{F}_{1}^{2 \ell}\left(\boldsymbol{\theta}^{00}\right) \mathbf{v}\right\|}{\left\|\mathcal{T}_{1}^{2 \ell}\left(\boldsymbol{\theta}^{00}\right) \mathbf{v}\right\|}
$$

is depicted in Figure 3.1.

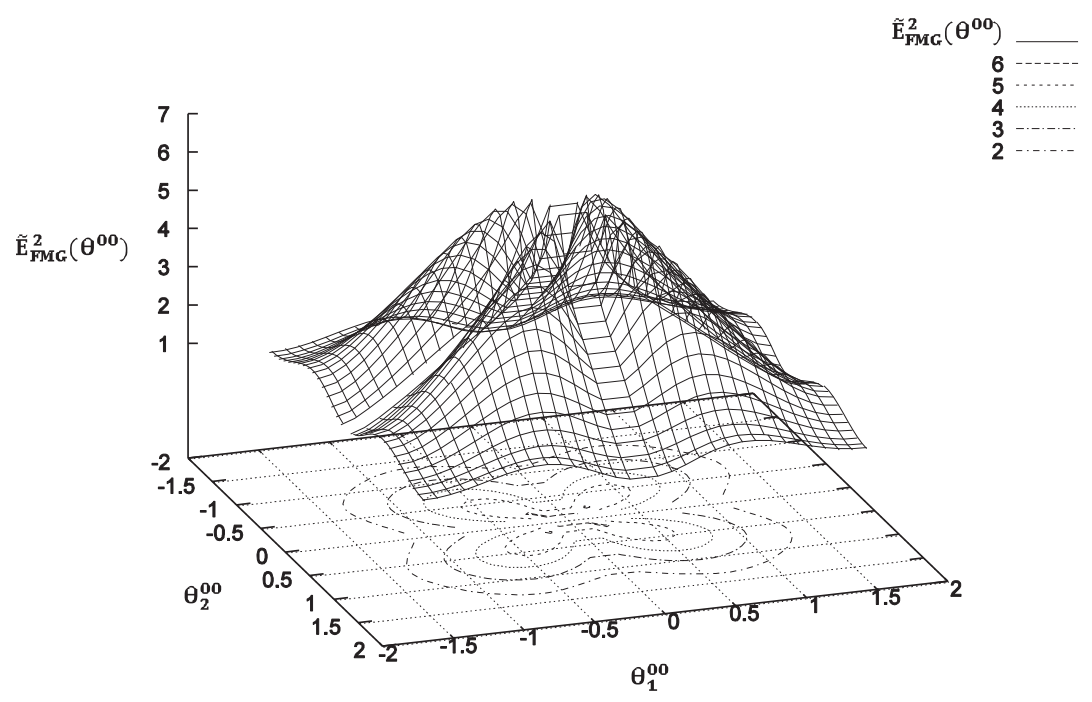
side.

FIG. 3.1. $\widetilde{E}_{F M G}^{2}\left(\boldsymbol{\theta}^{00}\right)$ for each low frequency $\boldsymbol{\theta}^{00}$, with injection used to restrict the right-hand

The maximum value, $\widetilde{E}_{F M G}^{2}$, is obtained for $\boldsymbol{\theta}^{00}=(-\pi / 16 h,-\pi / 16 h)$, and we can find the linear combination of the four harmonics associated with $\boldsymbol{\theta}^{00}$ yielding exactly the worst-case right-hand side that gives rise to the value predicted by the analysis. To this end, the eigenvector associated with the square of $\widetilde{E}_{F M G}^{2}$ is $\mathbf{w}=$ $(0.0558,0.7908,0.4310,0.4310)^{t}$, and, by calculating the product $\left(\mathcal{T}_{1}^{2 \ell}\left(\boldsymbol{\theta}^{00}\right)\right)^{-1} \mathbf{w}$, the corresponding vector $\mathbf{v}$ is determined. Consequently, the worst-case right-hand side reads:

$$
\begin{aligned}
f(x, y)= & 1.3356 e^{-i \pi x / 16 h} e^{-i \pi y / 16 h}+11.5324 e^{i 15 \pi x / 16 h} e^{i 15 \pi y / 16 h} \\
& +3.1872 e^{i 15 \pi x / 16 h} e^{-i \pi y / 16 h}+3.1872 e^{-i \pi x / 16 h} e^{i 15 \pi y / 16 h} .
\end{aligned}
$$

Next, we apply the corresponding two-level FMG algorithm to solve the discrete problem (3.15) on a fine grid of size $64 \times 64$, with right-hand side (3.16). The first row of Table 3.1 shows both relative errors $\left\|\mathcal{J}_{1} u-u_{1}\right\| /\|f\|$ and $\left\|\mathcal{J}_{1} u-u_{1}^{F M G}\right\| /\|f\|$, computed in the two-norm, and the ratio $\left\|\mathcal{J}_{1} u-u_{1}^{F M G}\right\| /\left\|\mathcal{J}_{1} u-u_{1}\right\|$, together with the value predicted by the two-level FMG analysis, $\widetilde{E}_{F M G}^{2}$. The predicted value matches the experimentally computed ratio, confirming the analysis.

Next, we perform the same experiment using the standard full-weighting operator for the restriction of the right-hand side, $J_{1}^{2}$. Analogous to the previous case, the values of $\widetilde{E}_{F M G}^{2}\left(\boldsymbol{\theta}^{00}\right)$ are depicted in Figure 3.2. 
TABLE 3.1

Results corresponding to the FMG analysis and numerical experiments for two different restriction operators for the right-hand side.

\begin{tabular}{|c|c|c|c|c|}
\cline { 2 - 5 } \multicolumn{1}{c|}{} & $\frac{\left\|\mathcal{J}_{1} u-u_{1}\right\|}{\|f\|}$ & $\frac{\left\|\mathcal{J}_{1} u-u_{1}^{F M G}\right\|}{\|f\|}$ & $\frac{\left\|\mathcal{J}_{1} u-u_{1}^{F M G}\right\|}{\left\|\mathcal{J}_{1} u-u_{1}\right\|}$ & $\widetilde{E}_{F M G}^{2}$ \\
\hline Injection & $1.9604 \times 10^{-5}$ & $1.2012 \times 10^{-4}$ & 6.127 & 6.127 \\
Full-weighting & $2.5738 \times 10^{-5}$ & $2.8235 \times 10^{-5}$ & 1.097 & 1.097 \\
\hline
\end{tabular}

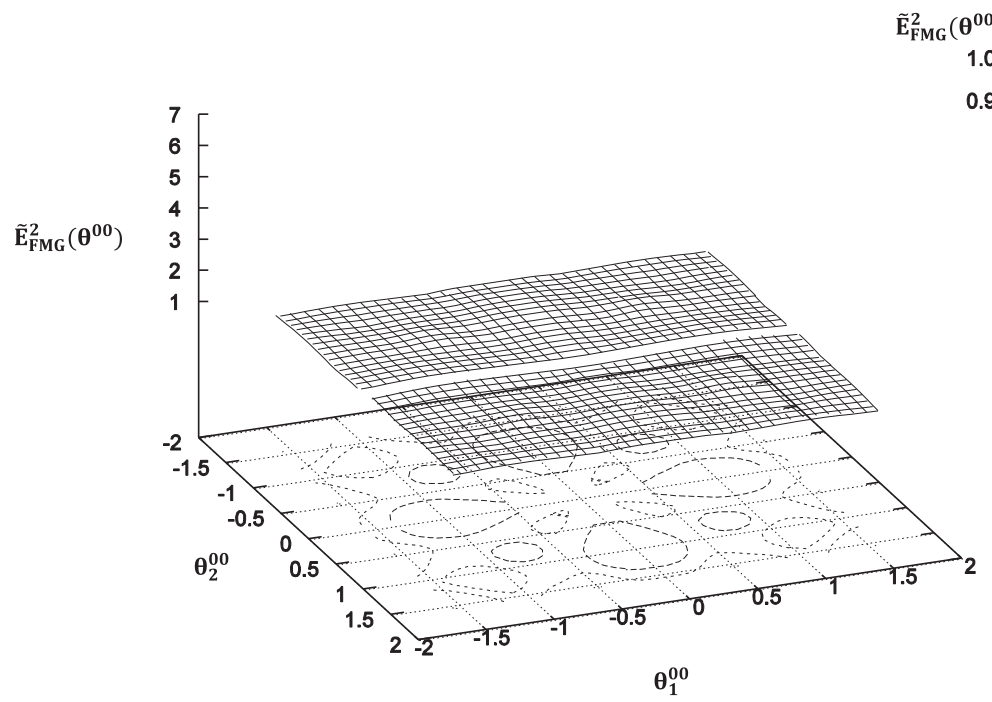

FIG. 3.2. $\widetilde{E}_{F M G}^{2}\left(\boldsymbol{\theta}^{00}\right)$ for each low frequency $\boldsymbol{\theta}^{00}$, with full-weighting restriction of the righthand side.

We observe an important improvement in the value predicted by the two-level FMG analysis, which now reads $\widetilde{E}_{F M G}^{2}=1.097$. This worst-case value is obtained for $\boldsymbol{\theta}^{00}=(-\pi / 32 h,-5 \pi / 16 h)$. We then compute as before the worst-case right-hand side, obtaining

$$
\begin{aligned}
f(x, y)= & -2.2784 e^{-i \pi x / 32 h} e^{-i 5 \pi y / 16 h}+0.0358 e^{i 31 \pi x / 32 h} e^{i 11 \pi y / 16 h} \\
& -0.0593 e^{i 31 \pi x / 32 h} e^{-i 5 \pi y / 16 h}-9.2077 e^{-i \pi x / 32 h} e^{i 11 \pi y / 16 h} .
\end{aligned}
$$

Again, we compare the worst-case ratio predicted by the analysis with the experimentally obtained ratio, by using the right-hand side given in (3.17) for problem (3.15). The results, shown in the second row of Table 3.1, once again match the analysis prediction. This test highlights the importance of applying local averaging of the right-hand side, rather than simple injection.

3.3. Extension to $k$-level FMG analysis. Due to the recursiveness of the operators involved in the FMG algorithm, $G_{k}^{\ell-k+1}$ and $M_{k}^{\ell-k+1}$, the two-level FMG analysis can be generalized to a $k$-level FMG analysis in order to estimate the corresponding $E_{F M G}^{k}$. It is well known that at least a three-level Fourier analysis is necessary to gain additional insight into the behavior of a multigrid algorithm, in particular, to distinguish between the performance of $\mathrm{V}$ - and $\mathrm{W}$-cycles, and the division between pre- and post-smoothing steps. Since the $k$-level operator $M_{1}^{k}$ arises in the 
definition of $\mathcal{F}_{1}^{k}$, it is natural to assume that with a $k$-level FMG analysis more insight into the performance of the FMG method can be obtained. If, for example, the FMG measures increase drastically with the number of levels, this is an indication of an unsatisfactory FMG algorithm which cannot achieve the required discretization-level accuracy.

To avoid excessive complication, in this section a detailed three-level FMG analysis is described. However, the generalization to a $k$-level FMG analysis can be made, and, in fact, some results obtained by a four-level FMG analysis are presented later.

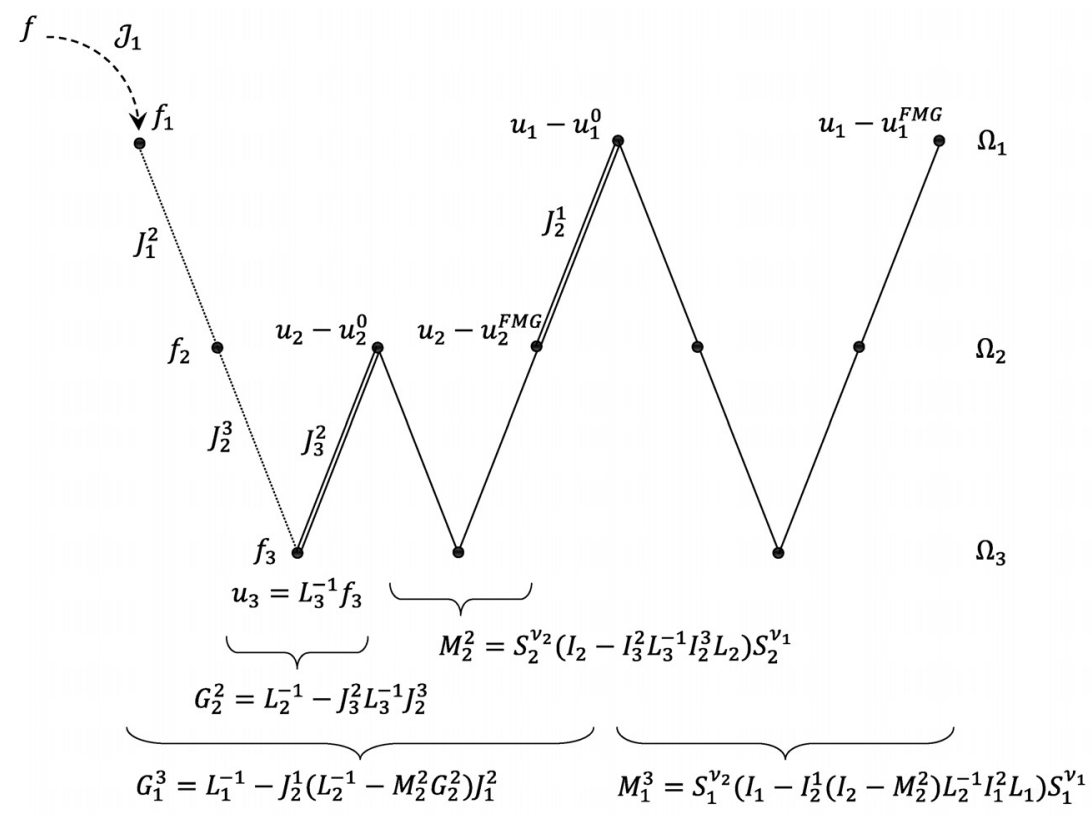

FIG. 3.3. Structure of the FMG iteration operator for three grid levels in the case $\nu=1$.

The aim of the three-level FMG Fourier analysis is to estimate the FMG accuracy measure $E_{F M G}^{3}$. The operators involved in the definition of $E_{F M G}^{3}$ are $\mathcal{T}_{1}$, and $\mathcal{F}_{1}^{3}:=$ $\mathcal{T}_{1}+\left(M_{1}^{3}\right)^{\nu} G_{1}^{3} \mathcal{J}_{1}$, where

$$
\begin{aligned}
M_{1}^{3} & =S_{1}^{\nu_{2}}\left(I_{1}-I_{2}^{1}\left(I_{2}-\left(M_{2}^{2}\right)^{\gamma}\right) L_{2}^{-1} I_{1}^{2} L_{1}\right) S_{1}^{\nu_{1}}, \\
G_{1}^{3} & =L_{1}^{-1}-J_{2}^{1}\left(L_{2}^{-1}-\left(M_{2}^{2}\right)^{\nu} G_{2}^{2}\right) J_{1}^{2},
\end{aligned}
$$

with $M_{2}^{2}$ and $G_{2}^{2}$ as defined in section 2 . These are illustrated, together with the other operators involved in the three-level FMG cycle, in Figure 3.3, where one V-cycle is applied. The initial algebraic error on the finest level is now given by

$$
u_{1}-u_{1}^{0}=G_{1}^{3} \mathcal{J}_{1} f
$$

and the final FMG algebraic error is

$$
u_{1}-u_{1}^{F M G}=\left(M_{1}^{3}\right)^{\nu} G_{1}^{3} \mathcal{J}_{1} f .
$$

Finally, the total error reads

$$
\mathcal{J}_{1} u-u_{1}^{F M G}=\left(\mathcal{J}_{1} u-u_{1}\right)+\left(u_{1}-u_{1}^{F M G}\right)=\mathcal{F}_{1}^{3} f .
$$


It is well known that the three-level operator $M_{1}^{3}$ couples 16 Fourier frequencies (see Figure 3.4), which define the subspaces of 4 h-harmonics (composed of four subspaces of $2 h$-harmonics $), \mathcal{E}_{1}^{16}\left(\boldsymbol{\theta}^{00}\right)=\mathcal{E}_{1}^{4}\left(\boldsymbol{\theta}_{00}^{00}\right) \cup \mathcal{E}_{1}^{4}\left(\boldsymbol{\theta}_{11}^{00}\right) \cup \mathcal{E}_{1}^{4}\left(\boldsymbol{\theta}_{10}^{00}\right) \cup \mathcal{E}_{1}^{4}\left(\boldsymbol{\theta}_{01}^{00}\right)$, $\boldsymbol{\theta}^{00} \in \tilde{\boldsymbol{\Theta}}_{3}=\boldsymbol{\Theta}_{3} \backslash \boldsymbol{\Psi}_{3}$, where $\boldsymbol{\Theta}_{3}=\left(-\pi / h_{3}, \pi / h_{3}\right] \times\left(-\pi / h_{3}, \pi / h_{3}\right]$, and $\boldsymbol{\Psi}_{3}=\left\{\boldsymbol{\theta}^{00} \in\right.$ $\boldsymbol{\Theta}_{3} \mid \operatorname{det}\left(\widetilde{\mathbf{L}}_{3}\left(4 \boldsymbol{\theta}^{00}\right)\right)=0$, or $\operatorname{det}\left(\widetilde{\mathbf{L}}_{2}\left(2 \boldsymbol{\theta}_{i j}^{00}\right)\right)=0$, or $\operatorname{det}\left(\widetilde{\mathbf{L}}_{1}\left(\boldsymbol{\theta}_{n m}^{i j}\right)\right)=0, i, j, n, m \in$ $\{0,1\}\}$, with

$$
\begin{aligned}
& \boldsymbol{\theta}_{i j}^{00}=\boldsymbol{\theta}^{00}-\left(i \pi \operatorname{sign}\left(\theta_{1}^{00}\right) / h_{2}, j \pi \operatorname{sign}\left(\theta_{2}^{00}\right) / h_{2}\right), \\
& \boldsymbol{\theta}_{n m}^{i j}=\boldsymbol{\theta}_{n m}^{00}-\left(i \pi \operatorname{sign}\left(\left(\theta_{n m}^{00}\right)_{1}\right) / h_{1}, j \pi \operatorname{sign}\left(\left(\theta_{n m}^{00}\right)_{2}\right) / h_{1}\right) .
\end{aligned}
$$

Note that the subspaces $\mathcal{E}_{1}^{16}\left(\boldsymbol{\theta}^{00}\right), \boldsymbol{\theta}^{00} \in \widetilde{\Theta}_{3}$, are invariant with respect to the operator $\left.M_{1}^{3} \cdot M_{1}^{3}\right|_{\mathcal{E}_{1}^{16}\left(\boldsymbol{\theta}^{00}\right)}$ can therefore be represented by a $16 \times 16$ eigenmatrix, denoted by $M_{1}^{3 \ell}\left(\boldsymbol{\theta}^{00}\right)$.
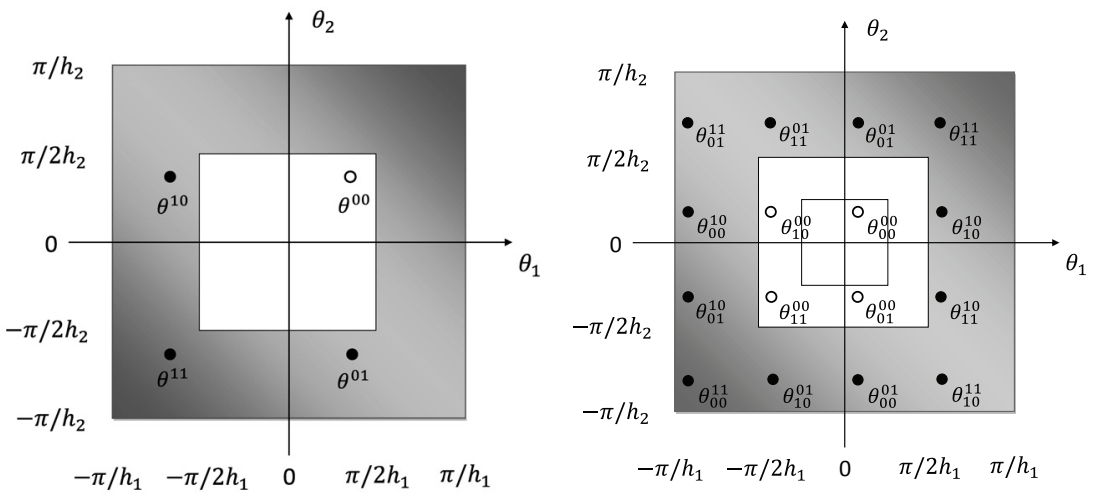

FIG. 3.4. Frequencies coupled by a two-level and a three-level iteration, which generate the space of $2 h$ - and $4 h$-harmonics.

Operator $G_{1}^{3}$ has the required invariant properties, $G_{1}^{3}: \mathcal{E}_{1}^{16}\left(\boldsymbol{\theta}^{00}\right) \rightarrow \mathcal{E}_{1}^{16}\left(\boldsymbol{\theta}^{00}\right)$, for each $\boldsymbol{\theta}^{00} \in \widetilde{\Theta}_{3}$, yielding a block-diagonal representation $\left.G_{1}^{3}\right|_{\mathcal{E}_{1}^{16}\left(\boldsymbol{\theta}^{00}\right)}=G_{1}^{3 \ell}\left(\boldsymbol{\theta}^{00}\right)$, given by

$G_{1}^{3 \ell}\left(\boldsymbol{\theta}^{00}\right)=\left(L_{1}^{3 \ell}\left(\boldsymbol{\theta}^{00}\right)\right)^{-1}-\left(J_{2}^{1}\right)^{3 \ell}\left(\boldsymbol{\theta}^{00}\right)\left(\left(L_{2}^{3 \ell}\left(\boldsymbol{\theta}^{00}\right)\right)^{-1}-\left(M_{2}^{3 \ell}\left(\boldsymbol{\theta}^{00}\right)\right)^{\nu} G_{2}^{3 \ell}\left(\boldsymbol{\theta}^{00}\right)\right)\left(J_{1}^{2}\right)^{3 \ell}\left(\boldsymbol{\theta}^{00}\right)$, where $M_{2}^{3 \ell}\left(\boldsymbol{\theta}^{00}\right)$ is the $4 \times 4$ eigenmatrix representation of the two-level operator $M_{2}^{2}$ on the subspace $\mathcal{E}_{2}^{4}\left(\boldsymbol{\theta}_{00}^{00}\right)=\operatorname{span}\left\{\varphi_{2}\left(\boldsymbol{\theta}_{i j}^{00}, \cdot\right) \mid i, j \in\{0,1\}, \boldsymbol{\theta}_{00}^{00} \in \Theta_{3}\right\}$, and

$$
\begin{aligned}
L_{1}^{3 \ell}\left(\boldsymbol{\theta}^{00}\right) & =\operatorname{bdiag}\left\{L_{1}^{2 \ell}\left(\boldsymbol{\theta}_{00}^{00}\right), L_{1}^{2 \ell}\left(\boldsymbol{\theta}_{11}^{00}\right), L_{1}^{2 \ell}\left(\boldsymbol{\theta}_{10}^{00}\right), L_{1}^{2 \ell}\left(\boldsymbol{\theta}_{01}^{00}\right)\right\} \in \mathbb{C}^{16 \times 16}, \\
\left(J_{2}^{1}\right)^{3 \ell}\left(\boldsymbol{\theta}^{00}\right) & =\operatorname{bdiag}\left\{\left(J_{2}^{1}\right)^{2 \ell}\left(\boldsymbol{\theta}_{00}^{00}\right),\left(J_{2}^{1}\right)^{2 \ell}\left(\boldsymbol{\theta}_{11}^{00}\right),\left(J_{2}^{1}\right)^{2 \ell}\left(\boldsymbol{\theta}_{10}^{00}\right),\left(J_{2}^{1}\right)^{2 \ell}\left(\boldsymbol{\theta}_{01}^{00}\right)\right\} \in \mathbb{C}^{16 \times 4}, \\
\left(J_{1}^{2}\right)^{3 \ell}\left(\boldsymbol{\theta}^{00}\right) & =\operatorname{bdiag}\left\{\left(J_{1}^{2}\right)^{2 \ell}\left(\boldsymbol{\theta}_{00}^{00}\right),\left(J_{1}^{2}\right)^{2 \ell}\left(\boldsymbol{\theta}_{11}^{00}\right),\left(J_{1}^{2}\right)^{2 \ell}\left(\boldsymbol{\theta}_{10}^{00}\right),\left(J_{1}^{2}\right)^{2 \ell}\left(\boldsymbol{\theta}_{01}^{00}\right)\right\} \in \mathbb{C}^{4 \times 16} \\
L_{2}^{3 \ell}\left(\boldsymbol{\theta}^{00}\right) & =\operatorname{diag}\left\{L_{2}^{2 \ell}\left(\boldsymbol{\theta}_{00}^{00}\right), L_{2}^{2 \ell}\left(\boldsymbol{\theta}_{11}^{00}\right), L_{2}^{2 \ell}\left(\boldsymbol{\theta}_{10}^{00}\right), L_{2}^{2 \ell}\left(\boldsymbol{\theta}_{01}^{00}\right)\right\} \in \mathbb{C}^{4 \times 4}, \\
G_{2}^{3 \ell}\left(\boldsymbol{\theta}^{00}\right) & =\left(L_{2}^{3 \ell}\left(\boldsymbol{\theta}^{00}\right)\right)^{-1}-\left(J_{3}^{2}\right)^{3 \ell}\left(\boldsymbol{\theta}^{00}\right)\left(L_{3}^{3 \ell}\left(\boldsymbol{\theta}^{00}\right)\right)^{-1}\left(J_{2}^{3}\right)^{3 \ell}\left(\boldsymbol{\theta}^{00}\right) \in \mathbb{C}^{4 \times 4},
\end{aligned}
$$

where bdiag denotes a block-diagonal matrix, and

$$
\begin{aligned}
\left(J_{3}^{2}\right)^{3 \ell}\left(\boldsymbol{\theta}^{00}\right) & =\left(\widetilde{J_{3}^{2}}\left(\boldsymbol{\theta}_{00}^{00}\right), \widetilde{J_{3}^{2}}\left(\boldsymbol{\theta}_{11}^{00}\right), \widetilde{J_{3}^{2}}\left(\boldsymbol{\theta}_{10}^{00}\right), \widetilde{J_{3}^{2}}\left(\boldsymbol{\theta}_{01}^{00}\right)\right)^{t} \in \mathbb{C}^{4 \times 1}, \\
\left(J_{2}^{3}\right)^{3 \ell}\left(\boldsymbol{\theta}^{00}\right) & =\left(\widetilde{J_{2}^{3}}\left(\boldsymbol{\theta}_{00}^{00}\right), \widetilde{J_{2}^{3}}\left(\boldsymbol{\theta}_{11}^{00}\right), \widetilde{J_{2}^{3}}\left(\boldsymbol{\theta}_{10}^{00}\right), \widetilde{J_{2}^{3}}\left(\boldsymbol{\theta}_{01}^{00}\right)\right) \in \mathbb{C}^{1 \times 4}, \\
L_{3}^{3 \ell}\left(\boldsymbol{\theta}^{00}\right) & =\widetilde{L_{3}}\left(4 \boldsymbol{\theta}^{00}\right) \in \mathbb{C}^{1 \times 1} .
\end{aligned}
$$

Copyright (c) by SIAM. Unauthorized reproduction of this article is prohibited. 
$\widetilde{\mathcal{T}}_{1}\left(\boldsymbol{\theta}_{n m}^{i j}\right)=\widetilde{\mathcal{L}}^{-1}\left(\boldsymbol{\theta}_{n m}^{i j}\right)-\widetilde{L}_{1}^{-1}\left(\boldsymbol{\theta}_{n m}^{i j}\right)$ is the Fourier symbol of operator $\mathcal{T}_{1}$, and because $\mathcal{T}_{1}: \mathcal{E}^{16}\left(\boldsymbol{\theta}^{00}\right) \rightarrow \mathcal{E}_{1}^{16}\left(\boldsymbol{\theta}^{00}\right)$, where $\mathcal{E}^{16}\left(\boldsymbol{\theta}^{00}\right)=\mathcal{E}^{4}\left(\boldsymbol{\theta}_{00}^{00}\right) \cup \mathcal{E}^{4}\left(\boldsymbol{\theta}_{11}^{00}\right) \cup \mathcal{E}^{4}\left(\boldsymbol{\theta}_{10}^{00}\right) \cup \mathcal{E}^{4}\left(\boldsymbol{\theta}_{01}^{00}\right)$, the matrix representation of $\mathcal{T}_{1}$ on these subspaces is

$$
\mathcal{T}_{1}^{3 \ell}\left(\boldsymbol{\theta}^{00}\right)=\operatorname{bdiag}\left\{\mathcal{T}_{1}^{2 \ell}\left(\boldsymbol{\theta}_{00}^{00}\right), \mathcal{T}_{1}^{2 \ell}\left(\boldsymbol{\theta}_{11}^{00}\right), \mathcal{T}_{1}^{2 \ell}\left(\boldsymbol{\theta}_{10}^{00}\right), \mathcal{T}_{1}^{2 \ell}\left(\boldsymbol{\theta}_{01}^{00}\right)\right\} \in \mathbb{C}^{16 \times 16} .
$$

Analogously, $\mathcal{F}_{1}^{3}: \mathcal{E}^{16}\left(\boldsymbol{\theta}^{00}\right) \rightarrow \mathcal{E}_{1}^{16}\left(\boldsymbol{\theta}^{00}\right)$, with

$$
\mathcal{F}_{1}^{3 \ell}\left(\boldsymbol{\theta}^{00}\right):=\mathcal{T}_{1}^{3 \ell}\left(\boldsymbol{\theta}^{00}\right)+\left(M_{1}^{3 \ell}\left(\boldsymbol{\theta}^{00}\right)\right)^{\nu} G_{1}^{3 \ell}\left(\boldsymbol{\theta}^{00}\right),
$$

is the $16 \times 16$ eigenmatrix representation of $\mathcal{F}_{1}^{3}$ with respect to the subspaces $\mathcal{E}^{16}\left(\boldsymbol{\theta}^{00}\right)$ and $\mathcal{E}_{1}^{16}\left(\boldsymbol{\theta}^{00}\right)$.

Finally, we can estimate the three-level FMG accuracy measure by the following expression

$$
\widetilde{E}_{F M G}^{3}=\sup _{\boldsymbol{\theta}^{00} \in \widetilde{\Theta}_{3}}\left\{\left\|\mathcal{F}_{1}^{3 \ell}\left(\boldsymbol{\theta}^{00}\right)\left(\mathcal{T}_{1}^{3 \ell}\left(\boldsymbol{\theta}^{00}\right)\right)^{-1}\right\| ; \operatorname{det}\left(\mathcal{T}_{1}^{3 \ell}\left(\boldsymbol{\theta}^{00}\right)\right) \neq 0\right\},
$$

and the $k$-level FMG accuracy measure, using a $k$-level FMG analysis, by

$$
\widetilde{E}_{F M G}^{k}=\sup _{\boldsymbol{\theta}^{00} \in \widetilde{\Theta}_{k}}\left\{\left\|\mathcal{F}_{1}^{k \ell}\left(\boldsymbol{\theta}^{00}\right)\left(\mathcal{T}_{1}^{k \ell}\left(\boldsymbol{\theta}^{00}\right)\right)^{-1}\right\| ; \operatorname{det}\left(\mathcal{T}_{1}^{k \ell}\left(\boldsymbol{\theta}^{00}\right)\right) \neq 0\right\},
$$

where $\mathcal{T}_{1}^{k \ell}, \mathcal{F}_{1}^{k \ell}$, are the $\left(4^{k-1} \times 4^{k-1}\right)$ eigenmatrix representations of operators $\mathcal{T}_{1}^{k}$, and $\mathcal{F}_{1}^{k}$, with respect to the subspaces $\mathcal{E}^{4^{k-1}}\left(\boldsymbol{\theta}^{00}\right)$ and $\mathcal{E}_{1}^{4^{k-1}}\left(\boldsymbol{\theta}^{00}\right)$. In the numerical experiments section some estimates of the $k$-level FMG accuracy measure, with $k=$ $2,3,4$, are presented.

3.3.1. Validation of the three-level FMG analysis. Similarly to the twolevel case, the three-level FMG analysis can be validated by means of the worstcase right-hand side analysis. Here, we briefly describe how to obtain the worst-case right-hand side. This particular right-hand side, $f$, is given by a linear combination of the Fourier components associated with the 16 frequencies coupled by the threelevel operators. The coefficients of this linear combination are given by a vector $\mathbf{v} \in$ $\mathbb{C}^{16}$, which can be obtained from $\mathbf{v}=\left(\mathcal{T}_{1}^{3 \ell}\left(\boldsymbol{\theta}^{00}\right)\right)^{-1} \mathbf{w}$, with $\mathbf{w}$ an eigenvector of the $16 \times 16$ matrix $W\left(\boldsymbol{\theta}^{00}\right)^{t} W\left(\boldsymbol{\theta}^{00}\right)$ associated with its maximum modulus eigenvalue, with $W\left(\boldsymbol{\theta}^{00}\right)=\mathcal{F}_{1}^{3 \ell}\left(\boldsymbol{\theta}^{00}\right)\left(\mathcal{T}_{1}^{3 \ell}\left(\boldsymbol{\theta}^{00}\right)\right)^{-1}$.

To validate the three-level FMG analysis based on this particular right-hand side, we again employ model problem (3.15). As in section 3.2.1, two different tests are performed, using either injection or full-weighting operators for $J_{1}^{2}$ and $J_{2}^{3}$. The other operators involved in the three-level FMG algorithm are the same as in the two-level case. In particular, the discrete operators, $L_{k}$, are the standard five-point discretizations. The initial solution on each grid is obtained by bilinear interpolation of the approximation from the next-coarser grid. For $I_{k}^{k+1}$ and $I_{k+1}^{k}$, full-weighting and bilinear interpolation are used, respectively, and the smoother is again damped Jacobi with relaxation parameter $\omega=0.8$. A single three-level V-cycle with one pre- and one post-smoothing step is performed in both experiments.

In the first test, where the injection operator is used for restriction of the righthand sides, the ratio computed by the three-level FMG analysis, with a grid of size $64 \times 64$ in the frequency space, is $\widetilde{E}_{F M G}^{3}=30.3709$, obtained with the frequency $\boldsymbol{\theta}^{00}=$ $(-\pi / 32 h,-\pi / 32 h)$ and its harmonics. Associated with $\boldsymbol{\theta}^{00}$ is a linear combination of the corresponding 16 harmonics, from which the worst-case right-hand side predicted 
by the analysis can be constructed. The same procedure as in the two-level case is followed, that is, vector $\mathbf{v}$ is calculated by the product $\left(\mathcal{T}_{1}^{3 \ell}\left(\boldsymbol{\theta}^{00}\right)\right)^{-1} \mathbf{w}$, where $\mathbf{w}$ is an eigenvector associated with the square of $\widetilde{E}_{F M G}^{3}$.

We apply the three-level FMG algorithm to solve the discrete problem (3.15) on a fine grid of size $64 \times 64$, with the worst case right-hand side. The first row of Table 3.2 shows both relative errors $\left\|\mathcal{J}_{1} u-u_{1}\right\| /\|f\|$ and $\left\|\mathcal{J}_{1} u-u_{1}^{F M G}\right\| /\|f\|$, computed in the two-norm, and the ratio $\left\|\mathcal{J}_{1} u-u_{1}^{F M G}\right\| /\left\|\mathcal{J}_{1} u-u_{1}\right\|$, together with the value predicted by the three-level FMG analysis, $\widetilde{E}_{F M G}^{3}$. Once again, we see a match with the analysis.

TABLE 3.2

Three-level results of the worst-case analysis computations and LFA analysis for two different restriction operators.

\begin{tabular}{|c|c|c|c|c|}
\cline { 2 - 5 } \multicolumn{1}{c|}{} & $\frac{\left\|\mathcal{J}_{1} u-u_{1}\right\|}{\|f\|}$ & $\frac{\left\|\mathcal{J}_{1} u-u_{1}^{F M G}\right\|}{\|f\|}$ & $\frac{\left\|\mathcal{J}_{1} u-u_{1}^{F M G}\right\|}{\left\|\mathcal{J}_{1} u-u_{1}\right\|}$ & $\widetilde{E}_{F M G}^{3}$ \\
\hline Injection & $2.006 \times 10^{-5}$ & $6.094 \times 10^{-4}$ & 30.371 & 30.371 \\
Full-weighting & $1.043 \times 10^{-5}$ & $3.749 \times 10^{-5}$ & 3.595 & 3.595 \\
\hline
\end{tabular}

Next, we repeat the experiment using full-weighting for the restriction of the righthand sides. The maximum value is now $\widetilde{E}_{F M G}^{3}=3.595$, and an important improvement is again observed. This value is obtained for a frequency $\boldsymbol{\theta}^{00}=(-\pi / 8 h,-\pi / 8 h)$, and in order to validate the predictions of the three-level FMG analysis for this case the worst-case right-hand side is again numerically computed. These results are shown in the second row of Table 3.2, once again demonstrating a match with the analysis.

4. Numerical experiments. To demonstrate the potential use of the $k$-level FMG analysis, we consider several problems and discretizations. The first test problem, the standard five-point discretization for the Laplace operator, serves to show how to determine the influence of various components of the FMG algorithm. For example, the importance of the type of cycle, the number of pre- and post-smoothing steps, the type of smoother, and the restriction for the right-hand side, are all evaluated. The remaining experiments test other PDEs and discretizations.

For the calculation of the estimates of the $k$-level FMG accuracy measure, $\widetilde{E}_{F M G}^{k}$, a $512 \times 512$ grid in frequency space is employed. The transfer operators are bilinear interpolation and full-weighted restriction, $I_{k+1}^{k}$ and $I_{k}^{k+1}$, respectively, and these remain fixed for all the experiments. Moreover, the discrete operator on each level of the hierarchy will be taken as the direct discretization of operator $\mathcal{L}$.

4.1. $\mathcal{O}\left(\boldsymbol{h}^{2}\right)$ Laplace discretization. The first example is the standard $\mathcal{O}\left(h^{2}\right)$ five-point finite difference discretization of the Laplace operator. First, we fix the restriction for the right-hand sides to be the full-weighting operator. Then, we compare the performance of different types of cycles and numbers of pre- and post-smoothing steps for three smoothers, plain Jacobi, damped Jacobi with relaxation parameter $\omega=0.8$, and red-black relaxation. For prolongation of the solution we use bilinear interpolation, and only a single $k$-level cycle is applied; that is, $\nu=1$. In Figure 4.1, the estimates of the two, three, and four-level FMG accuracy measures are depicted for $\mathrm{V}(1,0), \mathrm{V}(1,1), \mathrm{W}(1,0)$, and $\mathrm{W}(1,1)$, using red-black relaxation. It can be observed that, except for the $\mathrm{V}(1,0)$-cycle, all choices lead to an $\widetilde{E}_{F M G}^{k}$-factor independent of the number of levels in the analysis, $k$, which is clearly a desirable property. Furthermore, 
both $\mathrm{V}(1,1)$ - and $\mathrm{W}(1,1)$-cycles yield $k$-level FMG measures close to 1 , independently of $k$, indicating discretization level accuracy.

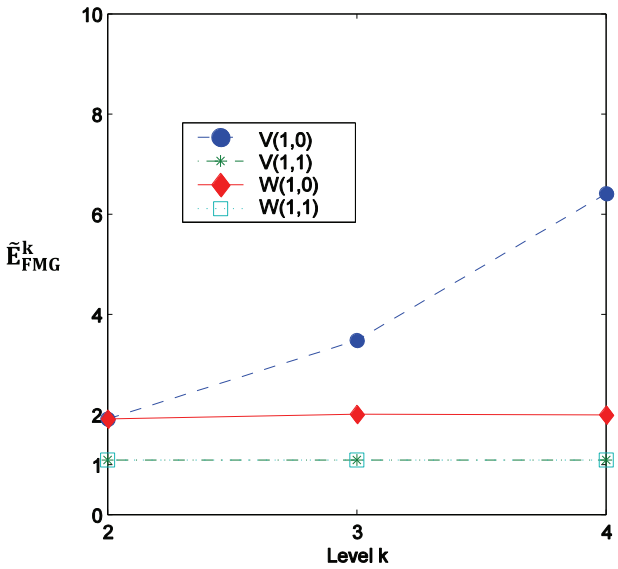

FIG. 4.1. $\widetilde{E}_{F M G}^{k}$ with $k=2,3,4$ for various types of cycles, with full-weighting restriction of the right-hand sides and red-black Gauss-Seidel smoothing.

Similarly, estimates for $\widetilde{E}_{F M G}^{k}$ with $k=2,3,4$ are depicted in Figure 4.2, with plain Jacobi (right-hand panel) or damped Jacobi with relaxation parameter $\omega=$ 0.8 (left-hand panel) in a $k$-level cycle. A rather surprising observation is that the performance of undamped Jacobi appears to be a satisfactory choice in FMG (even better than damped Jacobi) despite the well-known lack of the smoothing property of this scheme. For both smoothers, $k$-independent $\widetilde{E}_{F M G}^{k}$ factors close to 1 are obtained with the $\mathrm{W}(1,1)$-cycle. The $\mathrm{V}$-cycle, however, results in a deterioration of the FMG accuracy measure as the number of levels increases.
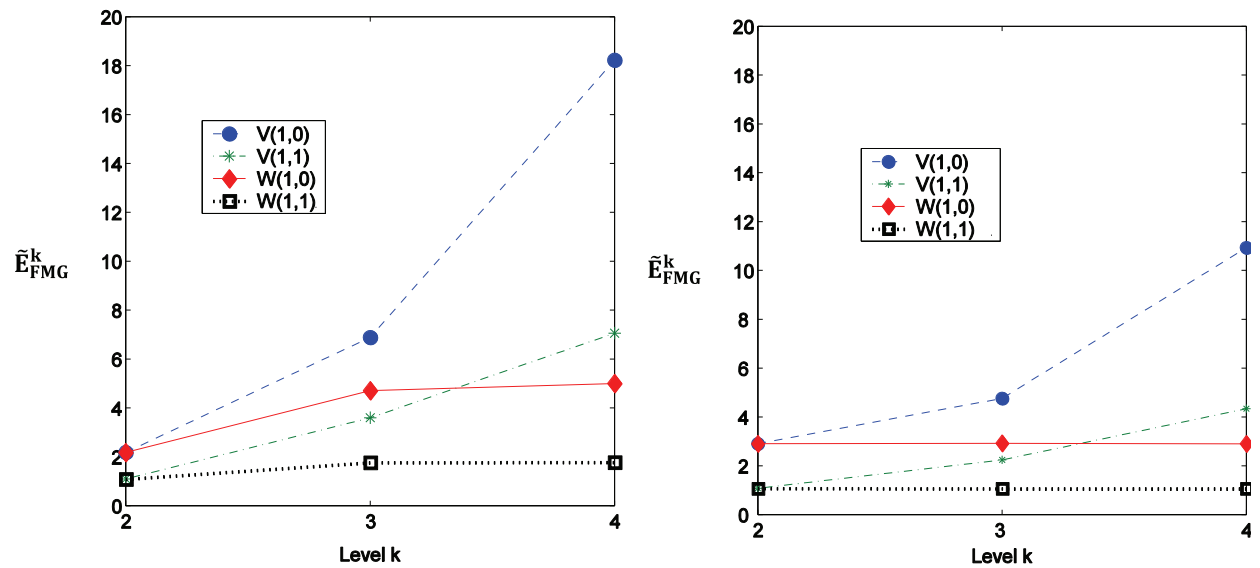

FIG. 4.2. $\widetilde{E}_{F M G}^{k}$ with $k=2,3,4$ for different types of cycles, with full-weighting restriction of the right-hand sides and a Jacobi smoother, with $\omega=0.8$ (left-hand panel) and with no damping (right-hand panel).

From the results, we conclude that one V(1,1)-cycle with a red-black Gauss-Seidel relaxation, combined with full-weighting restriction of the right-hand sides, provides a very efficient FMG method for the standard five-point discretization of the Laplace 
operator. The practical utility of this analysis is shown by means of some numerical experiments using different numbers of levels in FMG. The results obtained with the $k$-level FMG analysis with $k=2,3,4$ provide significant information about the performance of the FMG algorithm with an arbitrary number of levels.

As an example, we consider again problem (3.15) with a right-hand side consisting of a combination of sines associated with five different frequencies $\alpha_{i}=64 \pi / 2^{i}, i=$ $1, \ldots, 5$; that is,

$$
f(x, y)=\sum_{i=1}^{5} \sin \left(\alpha_{i} x\right) \sin \left(\alpha_{i} y\right) .
$$

We solve this problem by FMG, with the components described previously and a finest grid of size $128 \times 128$, for which the discretization error is $2.565 \times 10^{-6}$. In Table 4.1, for each of the three smoothers and various numbers of levels in the FMG algorithm, employing a $\mathrm{V}(1,0)$-cycle, the relative total error $\left\|\mathcal{J}_{1} u-u_{1}^{F M G}\right\| /\|f\|$ and the experimentally obtained ratio $\left\|\mathcal{J}_{1} u-u_{1}^{F M G}\right\| /\left\|\mathcal{J}_{1} u-u_{1}\right\|$ are shown. A deterioration of the experimental ratio is observed for all three smoothers as the number of levels in the FMG V(1,0)-cycle increases. These results confirm the trend from the two, three, and four FMG analysis from Figures 4.1 and 4.2.

TABLE 4.1

Experimentally computed ratios and total errors for various numbers of levels used in the FMG cycle, employing $V(1,0)$-cycles with three different smoothers.

\begin{tabular}{|c|c|c|c|c|c|c|}
\cline { 2 - 7 } \multicolumn{1}{c|}{} & \multicolumn{2}{c|}{ Jacobi } & \multicolumn{2}{c|}{$\omega$-Jacobi } & \multicolumn{2}{c|}{ Red-black } \\
\hline$\ell$ & ratio & error & ratio & error & ratio & error \\
\hline 2 & 0.925 & $2.373 \times 10^{-6}$ & 1.297 & $3.326 \times 10^{-6}$ & 0.928 & $2.379 \times 10^{-6}$ \\
3 & 2.079 & $5.331 \times 10^{-6}$ & 3.313 & $8.496 \times 10^{-6}$ & 1.679 & $4.306 \times 10^{-6}$ \\
4 & 4.496 & $1.153 \times 10^{-5}$ & 7.589 & $1.946 \times 10^{-5}$ & 3.241 & $8.313 \times 10^{-6}$ \\
5 & 8.383 & $2.150 \times 10^{-5}$ & 15.712 & $4.029 \times 10^{-5}$ & 5.533 & $1.419 \times 10^{-5}$ \\
6 & 14.256 & $3.656 \times 10^{-5}$ & 30.740 & $7.884 \times 10^{-5}$ & 8.565 & $2.197 \times 10^{-5}$ \\
\hline
\end{tabular}

Next, the same experiments are performed using FMG with W(1,1)-cycles; the corresponding results are given in Table 4.2. In this case, the experimentally computed ratios remain constant and close to 1 for all numbers of levels, independent of the smoother chosen. Furthermore, the discrete solution obtained with any of these approaches approximates the continuous solution to discretization level accuracy.

TABLE 4.2

Experimentally computed ratios and total errors for different numbers of levels used in the FMG algorithm with $W(1,1)$-cycles and three different smoothers.

\begin{tabular}{|c|c|c|c|c|c|c|}
\cline { 2 - 7 } \multicolumn{1}{c|}{} & \multicolumn{2}{c|}{ Jacobi } & \multicolumn{2}{c|}{$\omega$-Jacobi } & \multicolumn{2}{c|}{ Red-black } \\
\hline$\ell$ & ratio & error & ratio & error & ratio & error \\
\hline 2 & 0.722 & $1.851 \times 10^{-6}$ & 0.539 & $1.382 \times 10^{-6}$ & 0.776 & $1.991 \times 10^{-6}$ \\
3 & 0.735 & $1.884 \times 10^{-6}$ & 0.873 & $2.240 \times 10^{-6}$ & 0.744 & $1.909 \times 10^{-6}$ \\
4 & 0.706 & $1.810 \times 10^{-6}$ & 0.909 & $2.332 \times 10^{-6}$ & 0.740 & $1.899 \times 10^{-6}$ \\
5 & 0.703 & $1.803 \times 10^{-6}$ & 0.909 & $2.331 \times 10^{-6}$ & 0.740 & $1.899 \times 10^{-6}$ \\
6 & 0.703 & $1.803 \times 10^{-6}$ & 0.909 & $2.331 \times 10^{-6}$ & 0.740 & $1.899 \times 10^{-6}$ \\
\hline
\end{tabular}

Note in summary that the two-level convergence factors predicted by a basic local Fourier analysis tool are insufficient for seeing the deterioration of FMG using $\mathrm{V}(1,0)$-cycles, but the $k$-level analysis yields a practical and useful tool.

To complete the analysis for this problem, some results with injection for the restriction of the right-hand sides are reported. In Table 4.3 the estimates of the 
FMG accuracy measure for $k=2,3$, and 4 levels are shown for different types of cycles and numbers of pre- and post-smoothing steps for one and two cycles per level in the FMG method. When the right-hand sides on coarse levels are defined by injection, FMG V-cycles do not exhibit good performance, as for increasing values of $k$ the estimates $\widetilde{E}_{F M G}^{k}$ also increase. On the other hand, FMG W-cycles provide very satisfactory convergence results, even with one cycle: $\widetilde{E}_{F M G}^{k}$ values do not depend on the number of levels, and the values are sufficiently small. Results obtained by bicubic interpolation of the FMG solution are not shown here, as the corresponding factors are very similar to those obtained by bilinear interpolation.

TABLE 4.3

$\widetilde{E}_{F M G}^{k}$ values for $k=2,3,4$, obtained with injection for the restriction of the right-hand sides, bilinear interpolation for the solution in FMG, and different types of cycles and numbers of pre- and post-smoothing steps. One and two cycles within the FMG algorithm are considered.

\begin{tabular}{|c|c|c|c|c|c|c|}
\cline { 2 - 7 } \multicolumn{1}{c|}{} & \multicolumn{3}{c|}{ One cycle } & \multicolumn{3}{c|}{ Two cycles } \\
\cline { 2 - 7 } \multicolumn{1}{c|}{} & $\widetilde{E}_{F M G}^{2}$ & $\widetilde{E}_{F M G}^{3}$ & $\widetilde{E}_{F M G}^{4}$ & $\widetilde{E}_{F M G}^{2}$ & $\widetilde{E}_{F M G}^{3}$ & $\widetilde{E}_{F M G}^{4}$ \\
\hline $\mathrm{V}(1,1)$ & 4.437 & 20.751 & 86.497 & 1.106 & 1.755 & 5.208 \\
$\mathrm{~W}(1,1)$ & 4.437 & 5.013 & 5.102 & 1.106 & 1.111 & 1.111 \\
$\mathrm{~V}(2,1)$ & 4.408 & 20.711 & 86.276 & 1.069 & 1.476 & 3.666 \\
$\mathrm{~W}(2,1)$ & 4.408 & 4.736 & 4.789 & 1.069 & 1.072 & 1.072 \\
\hline
\end{tabular}

Remark. The examples presented have been selected to show that there is no immediate relation between the quality of the $k$-grid convergence factors of the multigrid cycle and the corresponding FMG accuracy measure. In fact, undamped Jacobi iteration provides very satisfactory FMG results, whereas an unsatisfactory iterative multigrid convergence is obtained. On the other hand, concerning the results in Table 4.3, we find fine results for $\mathrm{W}$-cycles and poor performance of V-cycles, where the corresponding multigrid convergence factors are both approximately 0.1 .

4.2. $\mathcal{O}\left(h^{4}\right)$ Laplace discretization. We next test the fourth-order "long stencil" discretization of the Laplace operator,

$$
L_{1} u_{1}=\frac{1}{12 h^{2}}\left[\begin{array}{ccccc} 
& 1 & & \\
& -16 & & \\
1 & -16 & 60 & -16 & 1 \\
& & -16 & & \\
& &
\end{array}\right] u_{1} .
$$

For this operator, we use injection for the restriction of the right-hand sides and a red-black smoother of Jacobi-type (see [18]) for the relaxation. For the intergrid transfer operators in the multigrid cycle, bilinear interpolation and full-weighting restriction are used, since we have observed that higher-order operators do not lead to any improvements in the FMG performance. The performances of two different interpolation operators for the FMG solution, bilinear and bicubic, are compared.

First, results obtained with an FMG method with a single cycle are presented in Table 4.4. W-cycles are considered since V-cycles display even worse convergence than the unsatisfactory results shown in this table. The $\widetilde{E}_{F M G}^{k}$ ratios are nearly constant with respect to the number of levels, but they are too large from a practical point of view. A significant improvement is obtained when bicubic interpolation is used instead, although the factors obtained then are also unsatisfactory. In both tests we 
found that an increase in the number of relaxation steps did not improve the estimated FMG accuracy.

TABLE 4.4

$\widetilde{E}_{F M G}^{k}$ values for $k=2,3,4$, obtained with injection for the restriction of the right-hand sides and two different interpolations for the FMG solution. A single cycle per level is performed in the FMG algorithm.

\begin{tabular}{|c|c|c|c|c|}
\cline { 3 - 5 } \multicolumn{2}{c|}{} & $\widetilde{E}_{F M G}^{2}$ & $\widetilde{E}_{F M G}^{3}$ & $\widetilde{E}_{F M G}^{4}$ \\
\hline \multirow{2}{*}{ Bicubic } & $\mathrm{W}(2,1)$ & 11.419 & 14.752 & 15.069 \\
& $\mathrm{~W}(2,2)$ & 11.240 & 13.369 & 13.553 \\
\hline \multirow{2}{*}{ Bilinear } & $\mathrm{W}(2,1)$ & 909.534 & 910.037 & 910.039 \\
& $\mathrm{~W}(2,2)$ & 907.201 & 907.721 & 907.723 \\
\hline
\end{tabular}

In the next test, we present in Figure 4.3 FMG analysis results with both interpolation operators for an FMG method using two cycles per FMG level. The $\widetilde{E}_{F M G}^{k}$ ratios for V-cycles increase with the number of levels. However, the FMG W-cycle factors remain constant with respect to increasing $k$. The FMG W(1,1)-cycle based on bicubic FMG interpolation appears most promising.
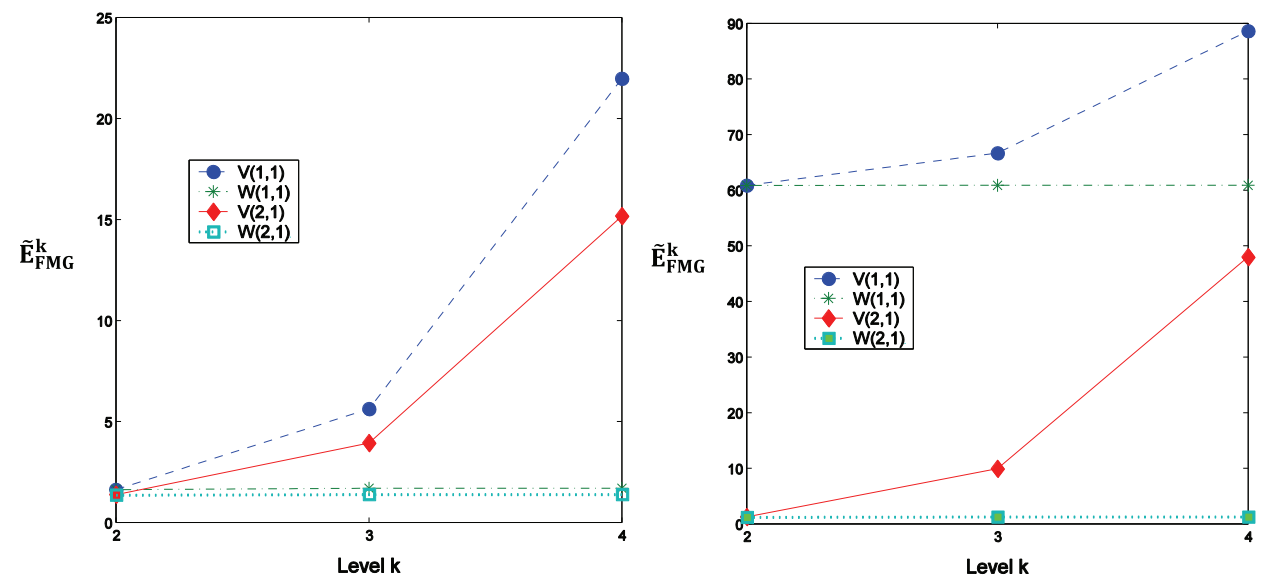

FIG. 4.3. $\widetilde{E}_{F M G}^{k}$ with $k=2,3,4$ for different types of cycles, with injection used to restrict the right-hand sides, red-black smoothing, and bilinear (right-hand panel) or bicubic (left-hand panel) interpolation.

Next, we provide some numerical results to assess the practicality of the LFA FMG measure. Problem (3.15) is again considered, with the right-hand side given by $f(x, y)=\sin (2 \pi x) \sin (2 \pi y)$. Two cycles per FMG level are applied, with injection and bilinear interpolation used for the FMG restriction and prolongation, respectively. FMG algorithms with V-and W-cycles are compared. In Table 4.5, the discretization and total errors obtained for different grid sizes and three different types of cycles are shown. For the $\mathrm{V}(1,1)$-cycle, for which the FMG analysis predicts increasing $\widetilde{E}_{F M G^{-}}^{k}$ values with respect to $k$, we observe that indeed the fourth-order accuracy is lost. For the $\mathrm{W}(1,1)$-cycle, the FMG accuracy estimates using $k=2,3,4$ levels were constant but too large $\left(\widetilde{E}_{F M G}^{k} \approx 60\right)$. Also here the fourth order is not preserved. However, with an FMG W(2,1)-cycle the reduction of the total error is satisfactory, and the 
expected fourth-order accuracy is achieved. This is predicted by the FMG analysis $\left(\widetilde{E}_{F M G}^{k}\right.$ remain constant and close to 1$)$.

TABLE 4.5

Experimentally computed discretization and total errors for different levels, using $V(1,1), W(1,1)$ and $W(2,1)$ cycles.

\begin{tabular}{|c|c|c|c|c|}
\hline \multirow{2}{*}{ grid size } & \multirow{2}{*}{$\frac{\left\|\mathcal{J}_{1} u-u_{1}\right\|}{\|f\|}$} & \multicolumn{3}{|c|}{$\frac{\left\|\mathcal{J}_{1} u-u_{1}^{F M G}\right\|}{\|f\|}$} \\
\cline { 3 - 5 } & & $\mathrm{V}(1,1)$ & $\mathrm{W}(1,1)$ & $\mathrm{W}(2,1)$ \\
\hline $32 \times 32$ & $2.085 \times 10^{-7}$ & $7.955 \times 10^{-6}$ & $2.514 \times 10^{-7}$ & $4.626 \times 10^{-8}$ \\
$64 \times 64$ & $1.306 \times 10^{-8}$ & $2.163 \times 10^{-6}$ & $4.096 \times 10^{-8}$ & $7.881 \times 10^{-9}$ \\
$128 \times 128$ & $8.169 \times 10^{-10}$ & $5.531 \times 10^{-7}$ & $4.942 \times 10^{-9}$ & $6.967 \times 10^{-10}$ \\
$256 \times 256$ & $5.106 \times 10^{-11}$ & $1.391 \times 10^{-7}$ & $8.765 \times 10^{-10}$ & $4.867 \times 10^{-11}$ \\
$512 \times 512$ & $3.192 \times 10^{-12}$ & $3.484 \times 10^{-8}$ & $1.965 \times 10^{-10}$ & $4.469 \times 10^{-12}$ \\
\hline
\end{tabular}

4.3. Biharmonic operator. We conclude the numerical experiments section by considering the discrete biharmonic operator, discretized by the following stencil:

$$
L_{1} u_{1}=\frac{1}{h^{4}}\left[\begin{array}{ccccc} 
& & 1 & & \\
& 2 & -8 & 2 & \\
1 & -8 & 20 & -8 & 1 \\
& 2 & -8 & 2 & \\
& & 1 & &
\end{array}\right] u_{1} .
$$

Here, we look for an efficient FMG algorithm for the resolution of this scalar problem, although it is also common to rewrite the biharmonic operator as a system of two coupled Poisson equations. As in the previous example, a red-black Jacobi smoother is used, and for the intergrid transfer operators in the multigrid cycle, bilinear interpolation and full-weighting restriction are chosen.

First, some results obtained by an FMG algorithm using injection for the restriction of the right-hand sides and bilinear prolongation for the interpolation of the FMG solutions are presented. The LFA estimates, $\widetilde{E}_{F M G}^{k}$ for $k=2,3$, 4, obtained with this FMG method are very large, giving rise to unsatisfactory numerical FMG results. In order to improve these values, the acceleration by weighting the relaxation scheme is pointed out in [2] as an interesting strategy for PDEs with higher-order derivatives. Optimal relaxation parameters for red-black Jacobi (determined in [11]) were found to be $\omega_{1}=1.25$ (pre-smoothing) and $\omega_{2}=0.7$ (post-smoothing) for the biharmonic operator. These relaxation parameters gave an impressive multigrid convergence improvement in [11], compared to the standard case $\omega_{1}=\omega_{2}=1$. As we will see, this significant improvement is also observed for FMG. The FMG accuracy measures with LFA for $k=2,3,4$ are presented in Table 4.6, for algorithms employing different numbers of cycles and pre- and post-smoothing steps. A significant improvement caused by the optimal relaxation parameters is observed, yielding FMG measures close to 1 and independent of $k$ for the FMG W $(2,1)$-cycle with $\nu=3 .^{2}$

\footnotetext{
${ }^{2}$ Note that FMG algorithms using only one cycle are not considered, since the estimates exceed values of $10^{4}$, nor are $\mathrm{V}$-cycles considered since the ratios provided by the analysis increase as $k$ gets larger.
}

Copyright $@$ by SIAM. Unauthorized reproduction of this article is prohibited. 
TABLE 4.6

$\widetilde{E}_{F M G}^{k}$ values for $k=2,3,4$, obtained with injection for the restriction of the right-hand sides and bilinear interpolation for the solution in FMG. Different FMG cycles: $W(1,1)$ and $W(3,2)$ with $\nu=2$ and $W(2,1)$ with $\nu=3$ are considered, for both $\omega_{1}=\omega_{2}=1.0$ and $\omega_{1}=1.25, \omega_{2}=0.7$.

\begin{tabular}{|c|c|c|c|c|}
\cline { 3 - 5 } \multicolumn{2}{c|}{} & $\widetilde{E}_{F M G}^{2}$ & $\widetilde{E}_{F M G}^{3}$ & $\widetilde{E}_{F M G}^{4}$ \\
\hline \multirow{2}{*}{$\mathrm{W}(1,1), \nu=2$} & $\omega_{1}=\omega_{2}=1.0$ & $1.616 \times 10^{7}$ & $1.406 \times 10^{7}$ & $1.411 \times 10^{7}$ \\
\cline { 2 - 5 } & $\omega_{1}=1.25, \omega_{2}=0.7$ & $4.293 \times 10^{3}$ & $4.297 \times 10^{3}$ & $4.297 \times 10^{3}$ \\
\hline \multirow{2}{*}{$\mathrm{W}(3,2), \nu=2$} & $\omega_{1}=\omega_{2}=1.0$ & $1.428 \times 10^{5}$ & $1.422 \times 10^{5}$ & $1.423 \times 10^{5}$ \\
\cline { 2 - 5 } & $\omega_{1}=1.25, \omega_{2}=0.7$ & 5.272 & 5.662 & 5.849 \\
\hline \multirow{2}{*}{$\mathrm{W}(2,1), \nu=3$} & $\omega_{1}=\omega_{2}=1.0$ & $5.722 \times 10^{4}$ & $2.847 \times 10^{4}$ & $2.860 \times 10^{4}$ \\
\cline { 2 - 5 } & $\omega_{1}=1.25, \omega_{2}=0.7$ & 1.262 & 1.465 & 1.471 \\
\hline
\end{tabular}

To test the influence of various components in the FMG algorithm, we consider the biharmonic model problem on a unit square domain with periodic boundary conditions and right-hand side $f(x, y)=\sin (2 \pi x) \sin (2 \pi y)$. Choosing, for example, an FMG algorithm based on $\mathrm{W}(1,1)$ and two cycles per FMG level, with $\omega_{1}=\omega_{2}=1$ in the relaxation, gives estimates of the FMG measure which are too large. This is confirmed in the corresponding column of Table 4.7, where the relative total error increases as the grid is refined. For a W(3,2)-cycle, however, with two cycles per FMG level and the optimal values of $\omega_{1}$ and $\omega_{2}$, the values of the FMG analysis are sufficiently small and independent of $k$, resulting in a satisfactory performance of the FMG algorithm. The FMG approximation is indeed of discretization accuracy, as displayed in the corresponding column of Table 4.7 .

TABLE 4.7

Experimentally computed discretization and total errors for different numbers of levels, using $W(1,1)$ and $W(3,2)$ cycles with two cycles per FMG level $(\nu=2)$, injection for the right-hand sides, and bilinear interpolation for the FMG solution, and also $W(2,1)$ cycles with two cycles per FMG level $(\nu=2)$ and high-order intergrid transfer operators.

\begin{tabular}{|c|c|c|c|c|}
\hline \multirow{2}{*}{ grid size } & \multirow{2}{*}{$\frac{\left\|\mathcal{J}_{1} u-u_{1}\right\|}{\|f\|}$} & \multicolumn{3}{|c|}{$\frac{\mathcal{J}_{1} u-u_{1}^{F M G} \|}{\|f\|}$} \\
\cline { 3 - 5 } & & $\mathrm{W}(1,1), \nu=2$ & $\mathrm{~W}(3,2), \nu=2$ & $\mathrm{~W}(2,1), \nu=2$ \\
\hline $64 \times 64$ & $2.579 \times 10^{-7}$ & $4.643 \times 10^{-7}$ & $2.579 \times 10^{-7}$ & $2.579 \times 10^{-7}$ \\
$128 \times 128$ & $6.443 \times 10^{-8}$ & $5.879 \times 10^{-7}$ & $6.443 \times 10^{-8}$ & $6.443 \times 10^{-8}$ \\
$256 \times 256$ & $1.611 \times 10^{-8}$ & $6.199 \times 10^{-7}$ & $1.611 \times 10^{-8}$ & $1.615 \times 10^{-8}$ \\
$512 \times 512$ & $4.027 \times 10^{-9}$ & $6.288 \times 10^{-7}$ & $6.220 \times 10^{-9}$ & $4.980 \times 10^{-9}$ \\
\hline
\end{tabular}

Finally, when high-order intergrid transfer operators are used in the FMG algorithm, in particular high-order restrictions for the right-hand side and bicubic interpolation of the FMG solution, the estimates predicted by the FMG analysis are significantly better than those presented in Tables 4.6 and 4.7 (2 columns). Again, the choice of the optimal relaxation parameters yields methods with improved performance. With one cycle per FMG level, for example, a constant value, $\widetilde{E}_{F M G}^{k}=3.05$, is obtained with an FMG W(2,1)-cycle and the optimal values for $\omega_{1}, \omega_{2}$. With two cycles per FMG level, the values of the FMG measure are close to 1 when W-cycles are used: An FMG W(2,1)-cycle, with two FMG cycles, gives $\widetilde{E}_{F M G}^{k}=1.178$ for $k=2,3,4$. The corresponding total errors on various grids are presented in the last 
column of Table 4.7. The reduction of the total error is very satisfactory, and the expected second-order accuracy is achieved.

5. Conclusions. One of the challenging tasks in the application of FMG is to determine a priori when the computed approximation has achieved the desired discretization accuracy. We deal with this problem in this paper by means of a local Fourier analysis technique for FMG algorithms. This technique gives us, a priori, insights into the performance of various FMG components. An FMG accuracy measure has been defined, which appears to be a highly satisfactory indicator for the accuracy achieved by FMG. Some Fourier analysis results are presented to confirm the theoretical estimates, and numerical experiments illustrate its practical utility.

Looking ahead, we would like to point out that the measure and the LFA developed may naturally be applied to other techniques and situations in which the residual may not always be an accurate reflection on the discretization accuracy. For example, it can be applied to obtain convergence estimates for classical techniques like defect-correction or double discretization schemes [3]. This is part of future research.

\section{REFERENCES}

[1] A. Bonzì, High-order discretization and multigrid solution of elliptic nonlinear constrained optimal control problems, J. Comput. Appl. Math., 200 (2007), pp. 67-85.

[2] A. Brandt, Multi-level adaptive solutions to boundary-value problems, Math. Comp., 31 (1977), pp. 333-390.

[3] A. Brandt, Guide to multigrid development, in Multigrid Methods Lectures Notes in Mathematics, vol. 960, W. Hackbusch and U. Trottenberg, eds., Springer, Berlin, 1982, pp. 220312.

[4] A. BRAndt, Rigourous quantitative analysis of multigrid I. Constant coefficients two level cycle with L2 norm, SIAM J. Numer. Anal., 31 (1994), pp. 1695-1730.

[5] F. J. Gaspar, J. L. Gracia, and F. J. Lisbona, Fourier analysis for multigrid methods on triangular grids, SIAM J. Sci. Comput., 31 (2009), pp. 2081-2102.

[6] W. Hackbusch, Multi-grid Methods and Applications, Springer, Berlin, 1985.

[7] P. W. Hemker, Lecture Notes on Defect Correction, Technical Report Lecture Notes, Centrum voor Wiskunde en Informatica (CWI), Amsterdam, 2001.

[8] P. W. Hemker, W. Hoffmann, and M. H. van RaAlte, Fourier two-level analysis for discontinuous Galerkin discretization with linear elements, Numer. Linear Algebra Appl., 11 (2004), pp. 473-491.

[9] L. KRonsuÖ And G. Dahlquist, On the design of nested iterations for elliptic difference equations, BIT, 11 (1971), pp. 63-71.

[10] L. Kronsjö, A note on the "nested iterations" method, BIT, 15 (1975), pp. 107-110.

[11] C. W. Oosterlee and R. Wienands, A genetic search for optimal multigrid components within a Fourier analysis setting, SIAM J. Sci Comput., 24 (2002), pp. 924-944.

[12] E. M. Stein, Harmonic Analysis: Real-Variable Methods, Orthogonality, and Oscillatory Integrals, Princeton University Press, New Jersey, 1993.

[13] R. Stevenson, On the Validity of Local Mode Analysis of Multi-grid Methods, Ph.D. thesis, Rijks Univ. Utrecht, The Netherlands, 1990.

[14] K. StüBen And U. Trottenberg, Multigrid methods: Fundamental algorithms, model problem analysis and applications, in Multigrid Methods, Lecture Notes in Math. 960, W. Hackbusch and U. Trottenberg, eds., Springer-Verlag, Berlin, 1982, pp. 1-176.

[15] U. Trottenberg, C. W. Oosterlee, and A. Schüller, Multigrid, Academic Press, New York, 2001.

[16] P. Wesseling, An Introduction to Multigrid Methods, John Wiley, Chichester, UK, 1992.

[17] R. Wienands, C. W. Oosterlee, and T. Washio, Fourier analysis of GMRES $(m)$ preconditioned by multigrid, SIAM J. Sci. Comput., 22 (2000), pp. 582-603.

[18] R. Wienands and W. Joppich, Practical Fourier Analysis for Multigrid Methods, Chapman and Hall/CRC Press, Boca Raton, FL, 2005.

Copyright (c) by SIAM. Unauthorized reproduction of this article is prohibited. 